Word count (excluding abstract, acknowledgements, references, figures and tables): 9,256

Short title/running head: PERFECTIONISM AND PSYCHOPATHOLOGY

\title{
The Relationship Between Perfectionism and Psychopathology: A Meta-Analysis
}

Keywords: perfectionism; psychopathology; meta-analysis; perfectionistic striving; perfectionistic concerns

Karina Limburg ${ }^{1,2 *}$, Hunna J. Watson ${ }^{1,3,4,5}$, Martin S. Hagger ${ }^{1}$, Sarah J. Egan ${ }^{1}$

${ }^{1}$ School of Psychology and Speech Pathology, Curtin University, Perth, Australia

${ }^{2}$ Clinic for Psychosomatic Medicine and Psychotherapy, University Hospital Klinikum rechts der Isar, Technische Universität München, Germany

${ }^{3}$ Eating Disorders Program, Specialised Child and Adolescent Mental Health Service, Perth, Australia

${ }^{4}$ School of Paediatrics and Child Health, The University of Western Australia, Perth, Australia

${ }^{5}$ Department of Psychiatry, University of North Carolina at Chapel Hill, United States

*Corresponding author: Karina Limburg, Clinic for Psychosomatic Medicine and Psychotherapy, University Hospital Klinikum rechts der Isar, Technische Universität München, Langerstraße 3, 81675 Munich, Germany, (e-mail: karina.limburg@tum.de, phone: +49894140 4397).

Full citation:

Limburg, K., Watson, H. J., Hagger, M. S., \& Egan, S. J. (2016). The relationship between perfectionism and psychopathology: A meta-analysis. Journal of Clinical Psychology. doi: $10.1002 /$ jclp. 22435 


\begin{abstract}
Objective: The clinical significance of two main dimensions of perfectionism (perfectionistic strivings and perfectionistic concerns) was examined via a meta-analysis of studies investigating perfectionism in the psychopathology literature.

Method: We investigated relationships between psychopathology outcomes (clinical diagnoses of depression, anxiety disorders, obsessive-compulsive disorder, and eating disorders, symptoms of these disorders, and outcomes related to psychopathology such as deliberate self-harm, suicidal ideation, and general distress) and each perfectionism dimension. The relationships were examined by evaluating (a) differences in the magnitude of association of the two perfectionism dimensions with psychopathology outcomes and (b) subscales of two common measures of perfectionism.
\end{abstract}

Results: A systematic literature search retrieved 284 relevant studies, resulting in 2,047 effect sizes that were analysed with meta-analysis and meta-regression while accounting for data dependencies.

Conclusions: Findings support the notion of perfectionism as a transdiagnostic factor by demonstrating that both dimensions are associated with various forms of psychopathology.

Keywords: perfectionism; psychopathology; meta-analysis; perfectionistic strivings; perfectionistic concerns 


\section{Introduction}

Perfectionism has a critical role in psychopathology. Many studies have linked perfectionism to affective disorders, anxiety disorders, obsessive-compulsive disorder (OCD), eating disorders, and other mental health problems. Egan, Wade and Shafran's (2011) narrative review concluded that perfectionism is a 'transdiagnostic' risk and maintaining factor for multiple psychological disorders.

Definitions of perfectionism centre on the pursuit of high standards and self-criticism over not meeting standards, and perfectionism has generally been conceptualised as multidimensional. The two most widely used measures of perfectionism are the Frost Multidimensional Perfectionism Scale (FMPS; Frost, Marten, Lahart, \& Rosenblate, 1990) and the Hewitt Multidimensional Perfectionism Scale (HMPS; Hewitt \& Flett, 1991b), each consisting of various subscales. Factor analysis of the scales typically results in a two-factor solution consisting of perfectionistic strivings and perfectionistic concerns (Bieling, Israeli, \& Antony, 2004; Frost, Heimberg, Holt, \& Mattia, 1993). Perfectionistic concerns has been suggested to be more strongly related to maladaptive outcomes such as negative affect, depression, stress, and anxiety, and perfectionistic strivings with adaptive outcomes, such as positive affect (Bieling, Israeli, et al., 2004; Frost et al., 1993). However, there is argument that perfectionistic strivings is also associated with maladaptive outcomes (e.g., Egan et al., 2011).

While the MPS scales have been widely used, demonstrated satisfactory reliability and validity, and have the advantage of enabling cross-study comparisons given their widespread use, there has been some criticism of the scales. One criticism has been that the FMPS subscale doubts about actions has substantive, non-trivial overlap with symptoms of OCD given the majority of items on the subscale were derived from a measure of OCD symptoms, the MOCI (Hodgson \& Rachman, 1977). Consequently it has been argued that the doubts about actions subscale primarily reflects checking symptoms of OCD, rather than 
perfectionism per se (Shafran \& Mansell, 2001). It has also been argued that the parental expectations and parental criticism subscales of the FMPS potentially confound the aetiological factors of perfectionism with the measurement of the construct given the subscale focuses on developmental aspects and the reporting of past experiences with parents (Rhéaume et al., 2000). Further, Shafran, Fairburn and Cooper (2003) argued that the widespread use of the MPS scales has led to reduced focus on understanding the maintaining aspects and the clinical relevance of perfectionism, which is why they proposed 'clinical perfectionism' referring to the pursuit of high standards despite negative consequences and basing self-worth on achievement (Fairburn, Cooper, \& Shafran, 2003a). This definition of clinical perfectionism has been used as a focus in the development of cognitive-behavioural treatments for perfectionism (see Egan, Wade, Shafran, \& Antony, 2014) which have evidence for efficacy (Lloyd, Schmidt, Khondoker, \& Tchanturia, 2015). An overview of the existing perfectionism scales and their categorisation into the two main domains can be seen in Table 1.

[Insert Table 1 here]

Our hypotheses are based on the accumulating evidence from studies that have shown dimensions of perfectionism are significantly higher in clinical samples with a range of disorders compared to controls, and associated with psychopathology in non-clinical samples.

The perfectionistic concerns dimension has consistently been shown to be significantly higher than controls in individuals with clinical disorders like depression (Enns, Cox, \& Borger, 2001; Hewitt \& Flett, 1991a; Huprich, Porcerelli, Keaschuk, Binienda, \& Engle, 2008; Norman, Davies, Nicholson, Cortese, \& Malla, 1998; Sassaroli et al., 2008), social anxiety disorder (Antony, Purdon, Huta, \& Swinson, 1998; Juster et al., 1996; Saboonchi, Lundh, \& Ost, 1999), panic disorder (Antony et al., 1998; Iketani et al., 2002), and OCD (Antony et al., 1998; Buhlmann, Etcoff, \& Wilhelm, 2008; Frost \& Steketee, 1997; Sassaroli et al., 2008). Perfectionistic concerns has also been linked to generalized anxiety 
disorder (Handley, Egan, Kane, \& Rees, 2014), and post-traumatic stress disorder (Egan, Hattaway, \& Kane, 2014). A smaller number of studies has found perfectionistic strivings to also be elevated in clinical disorders such as depression (Hewitt \& Flett, 1991a) and OCD (Antony et al., 1998; Buhlmann et al., 2008; Frost \& Steketee, 1997; Sassaroli et al., 2008), and associated with generalized anxiety disorder (Handley et al., 2014).

Perfectionism is a particularly strong risk and maintaining factor in eating disorders. Clinical perfectionism is one of several core maintaining mechanisms in Fairburn's transdiagnostic model of eating disorders (Fairburn et al., 2003a), which is the theoretical basis for cognitive-behavioral treatment for eating disorders. Perfectionism is also a central variable in the three-factor model of bulimia nervosa (Bardone-Cone, Abramson, Vohs, Heatherton, \& Joiner, 2006) and the cognitive-interpersonal model of anorexia nervosa (Schmidt \& Treasure, 2006). Individuals with anorexia nervosa and bulimia nervosa have significantly higher scores on perfectionistic strivings and perfectionistic concerns than controls (e.g., Cockell et al., 2002; Halmi et al., 2000; Lilenfeld et al., 2000; Moor, Vartanian, Touyz, \& Beumont, 2004; Sassaroli et al., 2008).

Similar patterns have been found in non-clinical populations, where perfectionistic concerns are positively correlated with depressive symptoms (Bieling, Israeli, et al., 2004; Enns, Cox, Sareen, \& Freeman, 2001; Graham et al., 2010) and trait anxiety (Gnilka, Ashby, $\&$ Noble, 2012). Further, perfectionistic strivings is positively related to depressive symptoms (Lombardo, Mallia, Battagliese, Grano, \& Violani, 2013). In non-clinical populations with symptoms of eating disorders, many subscales of perfectionism measures from both perfectionism dimensions have been found to be related to pathology (e.g., Brannan \& Petrie, 2008; Miller-Day \& Marks, 2006; Welch, Miller, Ghaderi, \& Vaillancourt, 2009).

There are also prospective studies which have linked perfectionism to the development of depression. These longitudinal studies are important as they give stronger evidence for the directional relation between perfectionism and depression since the majority 
of research is cross-sectional. For example, socially-prescribed perfectionism has been found to predict onset of depressive symptoms at follow-up (Békés et al., 2015; Hewitt, Flett, \& Ediger, 1996); and perfectionistic strivings predicts higher depressive symptoms at one-year follow-up in a clinical sample (Békés et al., 2015). Further, patients with clinical depression were followed over 3-year (Dunkley, Sanislow, Grilo, \& McGlashan, 2006) and 4-year (Dunkley, Sanislow, Grilo, \& McGlashan, 2009) follow-up periods and the perfectionism subscale of the Dysfunctional Attitudes Scale (DAS-SC; Weissman \& Beck, 1978), typically referred to as self-critical perfectionism, predicted increases in depressive symptoms. These findings have been further corroborated in a recent meta-analysis of 10 longitudinal studies of perfectionism and depression, where perfectionistic strivings and perfectionistic concerns had small, positive relationships with depressive symptoms at follow-up (Smith et al., 2016).

The existing body of research has some limitations. The concept of perfectionism and its measurement has been vigorously debated and has changed over time. The investigations of the associations between perfectionism and psychopathology have largely been disorderspecific, yet recently there has been growing interest in clinical psychology in transdiagnostic processes. Transdiagnostic processes are aspects of cognition or behaviour that contribute to maintenance of more than one psychological disorder (Harvey, Watkins, Mansell, \& Shafran, 2004) and have been referred to as being the points of intersection between personality and psychopathology (Rodriguez-Seijas, 2015). Thus, they may hold an important key to improving treatment efficacy. The findings from individual studies would benefit from contextualization to a transdiagnostic perspective. Although narrative reviews on the topic of perfectionism and psychopathology exist in eating disorders (Bardone-Cone et al., 2007) and across various disorders (Egan et al., 2011; Shafran \& Mansell, 2001), and single metaanalyses on treatment outcomes for perfectionism (Lloyd et al., 2015) and longitudinal studies of depression (Smith et al., 2016), there has been no quantitative synthesis of relations between perfectionism and psychopathological outcomes across disorders and symptoms 
using meta-analytic techniques. There have also been mixed study findings, with some studies reporting an association between perfectionistic strivings and psychopathology and other studies finding a null effect. Individual studies are limited in their ability to resolve these contradictions. To test the association between perfectionistic concerns and perfectionistic strivings with psychopathology, it is necessary to consider measures of each perfectionism domain.

\section{The Present Study}

The aim of this study was to investigate the relationship of perfectionistic strivings and perfectionistic concerns with psychopathology across studies using meta-analytic techniques. Specifically, we aimed to test whether the literature supports the hypothesis that perfectionistic concerns can be considered a transdiagnostic process across disorders in clinical samples and psychopathology in non-clinical samples as proposed by (Egan et al., 2011), and whether perfectionistic strivings is associated with psychopathology. The clinical relevance of understanding the link between perfectionism and psychopathology is that if perfectionism is found to be relevant across disorders (i.e., transdiagnostic), then it may be important to target in an attempt to reduce the symptoms of a range of disorders (Egan, Wade, et al., 2014). The second aim was to investigate the relative contribution of the subscales of the two most commonly used scales, the FMPS and HMPS, in the prediction of psychopathology, to address the question of whether there are certain subscales that show a stronger relationship to psychopathology than others. By identifying the scales that are most associated with psychopathology, it may be possible to recommend which subscales should be used to assess perfectionism in the context of psychopathology.

A meta-analysis of the extant literature may assist in providing a better understanding of the relationship between perfectionism and psychopathology across disorders and symptoms as it will provide bias-corrected estimates of the size and pattern of effects that cannot be gained from narrative reviews. Critically, we will examine the unique contribution 
of the perfectionism dimensions on psychopathology. Much of the research on perfectionism has examined zero-order effects of different perfectionism dimensions on outcomes. This does not account for the unique effects of the dimension on the outcome when accounting for effects of other perfectionism domains. Given that different dimensions of perfectionism have been shown to be significantly correlated, it is possible that overlap in the dimensions may give a misleading representation of the true effects of the dimensions. Due to this overlap, the zero-order effects of perfectionism dimensions will give a misleading, most likely inflated, representation of the true effects of the perfectionism dimensions. We will therefore test the unique effects of the perfectionism dimensions using meta-analytic path analysis of the weighted averaged correlations between perfectionistic dimensions and the psychopathological outcomes. The analysis will also permit the assessment of the degree of variability in effects across studies that cannot be attributed to the methodological artefacts corrected for in the analysis (i.e., sampling error). Identification of substantive heterogeneity in links between perfectionism and psychopathology will catalyse a search for key moderators to resolve the heterogeneity, a key goal of meta-analysis. We will evaluate the effects of potential moderators of the relationship between perfectionism and psychopathology outcomes, such as age, gender, and, in case of follow-up studies, the time between baseline assessment of perfectionism and the assessment of the outcome.

\section{Method}

\section{Search Strategy}

Several strategies were used to identify eligible studies. First, the databases ERIC, Embase, ISI Web of Science (Science Citation Index Expanded, Social Science Citation Index Expanded), Medline, PsycINFO, PsycARTICLES, and Scopus were searched for all years covered through to July 2013. Key words used were: perfectionism, mental health, outcome, behaviour/behavior, intervention, and psychopathology. Manual searches were 
conducted of reference lists from prior literature reviews and the electronic mailing list Perfectionism Network Mailing List was queried to identify studies that were accepted to a peer-reviewed journal, but not published at the time of the literature search. Active researchers in the field of perfectionism who had previously published two or more relevant articles on perfectionism and psychopathology were contacted to request additional citations.

\section{Relevant Outcomes}

The area of psychopathology contains a variety of outcomes and as such there was a need to classify them into appropriate, meaningful categories. Consequently, three broad categories of outcome were identified: clinical disorders, symptoms of disorders, and outcomes related to psychopathology. Through scanning the existing literature, four subcategories of frequently evaluated clinical disorders in the perfectionism literature were identified for inclusion in subsequent analyses: depression, anxiety disorders, OCD, and eating disorders (anorexia nervosa and bulimia nervosa). The relevant symptoms of disorders had to fall into one of the proposed disorder categories. Because studies reported various subsets of symptoms that represent the same symptom category, conceptually related symptoms were aggregated into analysable subcategories. For example, symptoms of social phobia that were measured in studies included fear of communication situation, fear of negative evaluation, shyness, social anxiety and social interaction anxiety, all of these were aggregated into the subcategory symptoms of social phobia. In addition, the OCD symptom compulsions was formed by integrating various reported compulsions, such as checking, cleaning, ordering, and washing. Additional clinical outcomes that cannot directly be related to a disorder such as suicidal ideation and self-harming behaviour were subsumed as outcomes related to psychopathology. This process of aggregation according to symptoms was done by the first author and the final author (Egan) who is an experienced Clinical Psychologist to ensure the classification was clinically meaningful. A summary of all analysed categories is presented in Figure 1. 
Insert Figure 1 here

\section{Inclusion and Exclusion Criteria}

The search strategy resulted in the identification of studies relevant to the relationship between perfectionism and different forms of psychopathology. Eligible studies were required to assess perfectionism using a validated self-report measure of trait perfectionism, and a relevant outcome (see Figure 1). All outcomes had to be assessed with validated measures. No restriction was placed on study characteristics regarding participant age, gender, race, or ethnicity; results from clinical and non-clinical samples were included; studies from any nation and any time period were considered relevant. Studies had to be printed or accepted in peer-reviewed journals. Dissertations or unpublished data were excluded to avoid the risk of retrieving duplicate effect sizes. Adequate detail of method, results, and data to calculate effect sizes had to be present for a study to be included. Eligible research designs included correlational studies and studies reporting a group comparison, for example, between a clinical and a non-clinical group. Reasons why studies were identified as not eligible were coded. The most common reasons for studies not being eligible are presented in Figure 2.

\section{Study Identification}

To determine study eligibility, titles and abstracts of all identified studies were examined by two independent judges that both held a bachelor's degree in psychology. If differing assessments occurred, those cases were discussed until consensus was reached. All remaining studies were then assessed in full text and coded by the first author.

\section{Coding Procedures}

The following data were coded for each of the eligible study reports: sample size, mean age of participants (years), proportion of female participants, sample type (i.e. clinical, non-clinical), diagnosis (if applicable), perfectionism measure, and effect sizes. Because 
some studies did not report the exact mean age of participants, it was estimated using valid indicators. For example, if data was reported on a sample of undergraduate students, it was estimated to be 19 years.

\section{Formation of Perfectionistic Strivings and Perfectionistic Concerns perfectionism}

Different views exist as to which subscales of which perfectionism measures should be subsumed under the two main perfectionism dimensions. For the purpose of this review, the formation of perfectionistic strivings and perfectionistic concerns follows the suggestions of (Stoeber \& Otto, 2006) who conducted a review of different conceptualisations of perfectionism and proposed recommendations on how to form the two dimensions based on theoretical considerations. In addition to the subscales of FMPS and HMPS, the authors considered the subscales of the Almost Perfect Scale - Revised (APS-R; Slaney, Rice, Mobley, Trippi, \& Ashby, 2001) and the Perfectionism Questionnaire (PQ; Rhéaume et al., 2000) in their classification.

Based on empirical evidence we determined a number of additional instruments to be included in the two dimensions, they can be found in Table 1. The decisions whether measures are valid for inclusion or exclusion of studies from the meta-analysis were made in an expert consensus process after considering the items of the various measures and aligning them to subscales of more established measures of perfectionism such as the FMPS and HMPS, and on the basis of correlations between these scales and psychopathology in reviews in the literature (Egan et al., 2011). Following Stoeber and Otto's (2006) recommendation, FMPS-Organization and HMPS-Other-Oriented Perfectionism have been omitted because of unclear findings as to whether these scales represent perfectionistic concerns or perfectionistic strivings. Furthermore, FMPS-Parental Expectations and Parental Criticism have been disregarded because these scales may not reflect core aspects of perfectionism but preceding factors that emerge during upbringing (Stoeber \& Otto, 2006). 


\section{Statistical Methods}

Effect size estimation procedure. Relevant primary studies reported either (a) zeroorder correlation coefficients, $r$, between perfectionism and the relevant outcome or (b) group comparisons between a clinical and a comparison group regarding perfectionism using parametric tests of difference such as $t$-tests or ANOVA models. Due to the aim of examining the relationship between perfectionism and an outcome and high number of studies reporting correlation coefficients, the zero-order correlation coefficient was selected as the effect size metric. Thus, the correlation coefficients reported in primary studies were extracted. Because the variance of the correlation coefficient depends on the correlation, standardization of the effect size using Fisher's $r$ to $Z$ transformation is recommended (Borenstein, Hedges, Higgins, \& Rothstein, 2009). All analyses were performed using the transformed values; the results were then back-transformed (Borenstein et al., 2009). When correlation coefficients were not reported, effect sizes were calculated from other statistics such as Cohen's $d$, the standardized mean difference score (Cohen, 1988). This was obtained through calculating the difference between the perfectionism means for the clinical and comparison conditions divided by the pooled standard deviation (Borenstein et al., 2009). The standardized mean difference $(d)$ was then converted into a correlation ( $r$ ) (Borenstein et al., 2009).

Statistical analysis. Two main sets of analyses were conducted in this study. The first set of analyses aimed to obtain weighted average effect sizes for the relationship between perfectionism and various outcomes related to psychopathology, thus gaining a general understanding of the size of the effect for the relations and the degree of heterogeneity associated with the effects. The second set of analyses aimed to further investigate the relationship by implementing meta-regression models.

An important issue that had to be considered in all analyses was the occurrence of data dependencies. Due to the fact that the majority of studies usually reported more than one outcome and these outcomes were often belonging to the same subcategory, there were many 
cases of nested effect sizes within studies. To address this concern, two meta-analytic methods were applied in the first set of analyses (the calculation of weighted average effect sizes). First, we used the conventional Hedges-Olkin random-effects model (Hedges \& Olkin, 1985) when effect sizes to be combined were not nested. Second, we used Hedges' robust variance estimation model (Hedges, Tipton, \& Johnson, 2010) when effect sizes to be combined contained nested effects. The robust variance estimation model is advantageous because it takes into account the within-study dependencies by introducing an estimate of the mean correlation $(\rho)$ between all pairs of nested effect sizes. This estimate is involved in the calculation of the between study sampling variance estimate, $\left(\tau^{2}\right)$. Because the robust variance estimator does not require information on the true correlation in the data, $\tau^{2}$ was estimated with $\rho=0.80$ in all analyses, as recommended by (Tanner-Smith, Wilson, \& Lipsey, 2013).

Weighting of the studies was conducted by calculating inverse-variance weights for all analyses. Heterogeneity in the effect sizes could be estimated via evaluation of the $\tau^{2}$ statistic in the context of the robust standard error estimation technique. To determine whether the observed heterogeneity is substantial or large, a prediction interval around the mean effect size $(\mu)$ can be calculated (after Black, 2009).

Second, to evaluate the relative contribution of the effects of perfectionism dimensions on psychopathological outcomes, we used the zero-order averaged weighted correlations between the dimensions and each psychopathology outcome as input into a metaanalytic path analysis. In each path analysis, the outcome of interest was regressed on to the perfectionism dimensions. The models were estimated using a maximum likelihood estimation method with the average sample size as the input sample size (Viswesvaran \& Ones, 1995). Given the number of analyses and sample sizes, we used a stringent probability level $(p<.01)$ to indicate a statistically significant effect and $95 \%$ confidence intervals of the parameter estimates to test whether the relative contribution of each perfectionism dimension differed. 
Finally, we applied nested meta-regression modelling to further investigate the relationship between perfectionism and psychopathology by comparing this relationship among various outcomes and sample types. In addition, to ensure sufficient statistical power, we set our criterion for the minimum number of primary studies per moderator group to ten, as recommended (Dalton \& Dalton, 2008). Only moderator variables evaluated in at least ten tests were considered in the meta-regression. To rule out type II errors, a sensitivity analysis was performed for each model after the meta-regression.

Tests for data censoring. Two forms of bias in the effect sizes, including variance that could be attributed to publication bias (Rosenthal, 1979) and funnel plots were visually inspected in order to detect asymmetry (Borenstein et al., 2009) and the fail-safe N-method was used to calculate the number of null results that would lead to a non-significant effect size if added to the analysis (Rosenthal, 1979). Egger's asymmetry test was also used to formally test for small-study biases that could be attributed to publication bias (Egger, Smith, Schneider, \& Minder, 1997; Hagger \& Chatzisarantis, 2014).

Statistical programs. The data were extracted and coded in Comprehensive MetaAnalysis V2.0 (CMA; Borenstein, Hedges, Higgins, \& Rothstein, 2005); the program was also used to standardise and convert amongst effect sizes. IBM SPSS Statistics V21.0 (IBM Corp., 2012) and MPlus version 7.31 analysis package (Muthén and Muthén, 2012) were used for the analyses.

\section{Results}

\section{Description of Studies}

The process of study selection is displayed in Figure 2. In sum, 284 studies containing 323 independent samples with effect size data and a total of 57,200 participants were included, 18 of these studies used a longitudinal design. The majority of participants were female $(74.0 \%)$ and mean sample age across studies was 25.06 years $(S D=8.13)$. The 
majority of primary studies $(65.1 \%)$ reported data from non-clinical samples. The psychological disorders most evaluated in relation to perfectionism were anxiety disorders (6.2\%), followed by eating disorders (4.6\%), OCD (3.9\%), and depression (1.6\%). The symptoms most evaluated were depressive symptoms (28.0\%), followed by symptoms of anxiety disorders $(19.8 \%)$, OCD (18.1\%), and eating disorders (12.0\%). Related outcomes including deliberate self-harm, suicidal behavior and ideation, and general psychological distress were evaluated in $5.8 \%$ of all tests. The majority of studies reported data from nonclinical samples and, thus, focused on symptoms of psychopathology rather than clinicallydiagnosed disorders. As a consequence, the focus for the current review is on disorder symptoms rather than clinically-diagnosed disorders. The perfectionism measure most utilized was the FMPS (48.1\%). The HMPS was used in $27.8 \%$ of all studies, followed by EDI-P (7.9\%), APS-R (5.3\%), OBQ-P (4.2\%), CAPS (2.1\%), DAS (1.9\%), and CPQ (0.7\%), with a small minority using other scales. Overall, 2,047 effect sizes across these outcomes were included in the analysis.

[Insert Figure 2 here]

\section{Weighted Average Effect Sizes}

To investigate the presence of heterogeneity, the preliminary meta-analysis did not distinguish between perfectionism dimensions or psychopathological outcome (i.e., clinical disorders, symptoms of disorders, outcomes related to psychopathology). All effect sizes from all studies were combined in a single analysis, and the weighted mean effect size was estimated using weighted random-effects analyses with robust variance estimates; this resulted in an overall effect size for the association between perfectionism and all psychopathological outcomes. The weighted average effect size was $0.26(n=2,047, k=323$, $p<0.001)$. The average $\tau^{2}$ of $0.05(S D=0.26, p<0.001)$ indicated the presence of additional heterogeneity in the effect size estimates unattributed to the methodological artefacts corrected for in the analysis. To determine whether this heterogeneity was substantial, the 
prediction interval was calculated to estimate the range in which a new estimated effect size would fall in $95 \%$ of new studies. The prediction interval was -0.54 to 3.0 , indicating a wide range in which a new effect size could fall, thus substantial heterogeneity was assumed. This finding confirmed our expectation, given that the literature refers to distinct influences of perfectionism in the context of different psychological disorders, symptoms, and outcomes related to psychopathology. Therefore, subsequent analyses of effect size exploring the moderation of the effect size by perfectionism dimensions and by separate psychopathological outcomes were justified.

\section{Small-Study Bias}

A funnel plot on the overall set of studies was investigated for asymmetry to test for small-study bias that might be indicative of publication bias (see Figure 3). Visual inspection of the plot appeared slightly asymmetric. More importantly, Egger's test for asymmetry based on the funnel plot indicated an absence of substantial bias.

\section{[Insert Figure 3 here]}

Perfectionism dimensions and psychopathology. We used weighted random-effects analyses with robust variance estimates to investigate the relationships between the two main perfectionism dimensions and psychological disorders, symptoms, and related outcomes. To draw basic conclusions about specific patterns of perfectionism for each outcome, effect sizes were pooled for the respective outcome and perfectionism dimension and evaluated separately. In addition to investigating the overall correlations between the two main perfectionism dimensions and psychopathology outcomes, another research objective was to assess the relative importance of perfectionistic concerns and perfectionistic striving. Previous studies have examined the overlap of the $95 \%$ confidence intervals (CIs) of the mean effect size for each outcome group (Tanner-Smith et al., 2013). However, this approach gives a misleading representation of the relative effects because it focuses on the zero-order correlations, which are, in essence, separate analyses. Instead, we conducted a series of meta- 
analytic path analyses in which each outcome was regressed on the two main perfectionism dimensions, perfectionistic concerns and perfectionistic strivings. The generalized model is depicted in Figure 4. Consistent with two-variable path-analytic models, the two predictors were correlated.

[Insert Table 2 and Figure 4 here]

The average weighted zero-order $(r)$ and unique $(\beta)$ effect sizes between perfectionism dimensions and psychopathological outcomes are presented in Table 2 . Regarding clinical disorders, there was evidence that both perfectionistic concerns and perfectionistic strivings were significantly related to depression, anxiety disorders, OCD, and bulimia nervosa. Only perfectionistic strivings was significantly related to anorexia nervosa and not perfectionistic concerns, and the variability in the latter effect size was substantial as illustrated by the wide confidence intervals that included the value of zero. Examination of the effect sizes from the path analyses provided detail on the relative contribution of each dimension in the prediction of psychopathological outcomes. For all outcomes, the unique effect $(\beta)$ for perfectionistic concerns was larger than the effect for strivings with no overlap in the confidence intervals of the effect size. Importantly, the effects for strivings was much smaller than the averaged zero-order correlation indicating that these effects were relatively trivial in comparison to the effects of concerns. Confidence intervals indicated that the effect for depression included the value of zero and the effect for OCD was approaching zero.

Regarding symptoms of disorders, the weighted average zero-order effect sizes were significant for the associations between the two main domains of perfectionism and various symptom outcomes, with the exception of the effect for social phobia symptoms and perfectionistic strivings. Examination of the unique effects from the path analyses revealed that the effects for perfectionistic concerns were substantially larger for many of the outcomes including depressive symptoms, anxiety, social phobia symptoms, worry, OCD symptoms, obsessive beliefs, global eating pathology, binge eating, and body dissatisfaction. 
For these outcomes, the CIs revealed significant differences in the size of the effects for perfectionistic concerns relative to perfectionistic strivings. In contrast, there was overlap in the CIs for the effect sizes for perfectionism dimensions on dietary restraint, drive for thinness, and thin-ideal internalisation. The effect for perfectionistic concerns and perfectionistic strivings were no different in the magnitude of the effects indicating a relatively equal contribution to explaining variance in eating disorder outcomes.

Regarding outcomes related to psychopathology, the perfectionism dimensions were significantly related to suicidal ideation and general psychological distress. The unique effects from the path analysis and their confidence intervals indicated significantly larger effects for perfectionistic concerns relative to perfectionistic strivings for both outcomes with the effects for strivings not significant or relatively trivial in size.

Overall, these data support the overall hypothesis of relations between perfectionism and psychopathology. Specifically, perfectionistic concerns and perfectionistic strivings were both significantly associated with a range of different psychological disorders including symptoms of psychological disorders and related outcomes within clinical and community samples. However, tests of the unique effects of the specific perfectionism dimensions revealed that perfectionistic concerns had the larger effect for most outcomes, the only exceptions were for outcomes related to eating disorders where concerns and strivings contributed approximately equally to explaining variance.

Perfectionism measures and psychopathology. The second aim also concerned the evaluation of each outcome in relation to perfectionism but was concerned with the two most commonly used perfectionism scales, the FMPS and HMPS. The purpose was to investigate whether certain perfectionism subscales show a stronger relationship with some disorders, symptoms, or related outcomes than others. As expected, average effect sizes revealed higher overall correlations on scales previously found to measure perfectionistic concerns, such as FMPS-concern over mistakes, doubts about actions, and HMPS-socially prescribed 
perfectionism than on scales previously found to measure perfectionistic strivings such as FMPS-personal standards and HMPS-self-oriented perfectionism for depression, anxiety disorders, and OCD. The same tendency was observed for anorexia nervosa, however the differences between magnitudes of correlation coefficients were smaller. Regarding the remaining scales of the two measures, worthy of note was a significant positive overall correlation for FMPS-organisation and anorexia nervosa. Further, the combined dimension of the parenting related subscales of the FMPS, Parental expectations and Parental criticism, showed significant positive (but small) overall correlations with anxiety disorders and OCD. The results are presented in Table 3. In order to examine heterogeneity due to betweenstudies variability, we evaluated various indicators: In case of dependent effect sizes, we examined the $\tau^{2}$ - statistic as a between study sampling variance estimate as it involves an estimate of the mean correlation $(\rho)$ between all pairs of nested effect sizes. In case of independent effect sizes, we evaluated the $Q$-statistic which informs about the presence of heterogeneity and the $I^{2}$ index which assesses the degree of heterogeneity. These values indicated substantial heterogeneity which can likely be due to extraneous moderator variables beyond the artefacts corrected for that may account for the variation in the magnitude of the correlation between perfectionism and outcome. Those moderator variables can be identified by meta-regression analyses.

In sum, various scales of both measures were differentially related to the different outcomes, with most scales previously found to measure perfectionistic concerns showing a stronger relationship to psychopathology than those previously found to measure perfectionistic strivings. Nevertheless, significant findings for the association between subscales measuring perfectionistic strivings (FMPS-personal Standards, FMPS-organisation, HMPS-self-oriented perfectionism) and psychopathology were found, indicating that in contrast to the view of previous authors (e.g., Stoeber \& Otto, 2006), perfectionistic strivings may also play a role in some forms of psychopathology. 
[Insert Table 3 here]

\section{Meta-Regression Analyses}

To examine the influences that may have led to the observed heterogeneity in the effect sizes for perfectionism dimensions on psychopathology outcomes, meta-regression analyses were implemented. This procedure was used to explain variance in the average weighted effect size of perfectionism and psychopathology with perfectionism dimensions and sample types as moderators. Specifically, the average weighted correlation between all perfectionism dimensions and outcomes was predicted in a series of nested meta-regression models with perfectionism dimension and sample type as predictors. Each meta-regression model aimed at answering a specific research question. The moderators were determined by dummy-coded contrast variables representing membership of the moderator groups. For moderators with more than two categories, a reference category had to be determined. The reference category was determined as the characteristic with the largest number of effect sizes. The first regression model aimed to predict the overall effect of perfectionism on all psychopathological outcomes controlling for study characteristics. The perfectionism dimensions (perfectionistic concerns, perfectionistic strivings, FMPS-concerns over mistakes, FMPS-doubts about actions, HMPS-socially prescribed perfectionism, FMPS-personal standards, HMPS-self-oriented perfectionism, FMPS-parental expectations and criticism, FMPS-organisation, HMPS-other-oriented perfectionism) and study characteristics (age, gender, time between baseline assessment of perfectionism and follow-up assessment of outcome) were the moderator variables. The reference category for the dummy variable perfectionism was determined to be perfectionistic concerns. Perfectionistic strivings and the remaining subscales measuring different aspects of perfectionism were compared with the reference category by evaluating the polarity of their coefficient.

The results are presented in Table 4. Perfectionistic strivings as well as HMPS-OtherOriented Perfectionism accounted for a significant amount of variance in effect sizes, both 
associated with a significantly smaller effect compared to perfectionistic concerns. No other perfectionism dimension or study characteristic yielded a significant effect.

[Insert Table 4 here]

The second set of meta-regression models was implemented to evaluate possible moderators as well as to investigate the subscales of FMPS and HMPS in specific sample types (clinical vs. non-clinical). Two meta-regression models were calculated, one restricted to clinical samples, the other restricted to non-clinical samples (see Table 5). Both models aimed to predict the overall effect of perfectionism on all psychopathological outcomes controlling for study characteristics in the respective sample type (clinical vs. non-clinical). The reference category for the dummy-coded perfectionism dimension moderator variable was overall perfectionistic concerns in both models. In the moderator analysis limited to clinical samples, HMPS-socially prescribed perfectionism accounted for a significant amount of variance in the effect size; it was associated with a significantly higher effect compared to perfectionistic concerns. The analysis restricted to non-clinical samples yielded significant negative regression coefficients for perfectionistic strivings and HMPS-other-oriented perfectionism, indicating a weaker link of psychopathology to these two domains than to perfectionistic concerns in non-clinical samples. It is important to note that fewer moderators could be evaluated in the clinical group compared to non-clinical group because there were fewer studies on clinical samples. This fact may serve to explain the differences between the two groups. For all models of the meta-regression procedures, no additional perfectionism dimension or study characteristic accounted for a significant amount of variance in effect sizes.

\section{[Insert Table 5 here]}

Finally to evaluate the role of the dimensions of perfectionism in the context of existing specific psychological disorders, one additional set of meta-regression models was computed. This implied one additional meta-regression per disorder, all models aimed to 
predict the overall effect of perfectionism on psychological disorders controlling for study characteristics. The only exception was the analysis for depression which contained fewer than ten tests so was deemed inappropriate to conduct an analysis due to the small sample size. Anorexia nervosa and bulimia nervosa were subsumed into a single "eating disorders" category due to the low numbers of tests for each disorder in the sample of studies. The reference category in each subset was overall perfectionistic concerns for the dummy coded variable perfectionism. Results are depicted in Table 6. Perfectionistic strivings, FMPSconcern over mistakes, and FMPS-organisation accounted for a significant amount of variance in anxiety disorders, with perfectionistic strivings and FMPS-organisation exhibiting negative effects and FMPS-concern over mistakes exhibiting positive effects compared to perfectionistic concerns. For OCD, the association of FMPS-concerns over mistakes, FMPSorganisation, and age to OCD was significantly lower than the correlation between perfectionistic concerns and OCD while the association between FMPS-doubts about actions and OCD was significantly higher. Among eating disorders, none of the perfectionism dimensions or study characteristics accounted for a significant amount of variance. ${ }^{1}$

\section{[Insert Table 6 here]}

To determine whether the chosen reference category had an impact on the findings, sensitivity analyses were conducted by (1) varying the reference categories in each model and (2) not controlling for the two broad perfectionism dimensions perfectionistic concerns and perfectionistic strivings. These analyses had no major impact on the findings, i.e., the direction of relationship and the statistical significance remained unchanged, so the interpretations of the findings are based on the results presented above.

\footnotetext{
${ }^{1}$ In addition to the meta-regression analyses presented here, we also conducted nested meta-regression models predicting effect sizes restricted to studies reporting on perfectionistic striving vs. perfectionistic concerns. However, these analyses did not yield any meaningful results and are thus not presented here.
} 


\section{Discussion}

The purpose of this meta-analysis was to investigate the relationships between perfectionism domains and psychopathology, the relative contribution of the subscales of FMPS and HMPS in the prediction of psychopathology, and to examine the effects of candidate moderators of the effects of perfectionism dimensions on psychopathological outcomes.

\section{Perfectionistic concerns vs. perfectionistic strivings in relation to psychopathology.}

The main finding was that both dimensions of perfectionism were associated with psychopathology outcomes across studies. In the majority of outcomes when the results of the path analysis are considered, perfectionistic strivings was less related to psychopathology than perfectionistic concerns, particularly in non-clinical populations. This finding supports the view of previous authors (e.g., Stoeber \& Otto, 2006). Amongst the investigated clinical disorders only eating disorders were an exception as both dimensions were strongly related to pathology as shown by the examination of weighted averaged correlation coefficients and unique effects. Furthermore, the two dimensions of perfectionism were overall positively correlated. This finding is important for theory as it indicates substantial overlap in the dimensions and indicates that zero-order correlations between these dimensions and outcomes may provide a misleading representation of the strength and pattern of effects. It highlights the need to account for unique effects of these constructs when predicting outcomes. The findings are also important for interventions and indicate that although both components of perfectionism should be targeted for outcomes relating to eating disorders, the focus for reducing symptoms of OCD, anxiety disorders and depression should be on perfectionistic concerns as this dimensions seems to contribute most in explaining variance in these psychopathological outcomes.

The positive associations between the perfectionism dimensions and outcomes across all domains of psychopathology are consistent with findings that perfectionism is not specific 
to certain disorders or symptoms (e.g., Frost \& Steketee, 1997; Rhéaume, Freeston, Dugas, Letarte, \& Ladouceur, 1995) and is a transdiagnostic process (Egan et al., 2011). Investigating the subscales of FMPS and HMPS.

Regarding the particular subscales of FMPS and HMPS in the context of psychopathology, several findings were observed. There were higher scores on scales which load on perfectionistic concerns, including FMPS-concern over mistakes, FMPS-doubts about actions, and HMPS-socially prescribed perfectionism than on scales loading on perfectionistic strivings such as FMPS-personal standards and HMPS-self-oriented perfectionism for depression, anxiety disorders, and OCD, supporting previous research (Bieling, Israeli, et al., 2004; Frost et al., 1993). However, this finding was not confirmed in the meta-regression, likely due to the fact that scores on some subscales were not reported in a sufficient number of scales so that they could not be evaluated in the meta-regression. To draw final conclusions in a meta-regression analysis, more studies reporting the effects of the different subscales of the two measures in the context of the particular disorders are needed.

For depression, the tendency of higher scores on HMPS-self-oriented perfectionism compared to FMPS-personal standards was revealed, consistent with research reporting high scores on HMPS-self-oriented perfectionism in depression (Hewitt \& Flett, 1991a) and low scores on FMPS-personal standards (Lombardo et al., 2013). This suggests that the assessment of perfectionism in the context of depression may concentrate on HMPS-selforiented perfectionism instead of FMPS-personal standards. This conclusion is drawn with caution however as depression was not evaluated in a sufficient number of studies to conduct a meta-regression restricted to effect sizes regarding depression. The meta-regression analyses restricted to eating disorders revealed a tendency for scores on FMPS-concern over mistakes to be lower in eating disorders compared to perfectionistic concerns, indicating that the latter combination of scales may be more suitable to assess perfectionism in eating disorders instead of single subscales like FMPS-concern over mistakes. Further, amongst 
OCD, FMPS-doubts about actions tended to be highly correlated to the presence of OCD as hypothesized, supporting previous findings of pronounced scores on FMPS-doubts about actions in OCD (Antony et al., 1998; Frost \& Steketee, 1997). This is not surprising given that some items which comprise doubts about actions were taken from a measure of OCD symptoms, thus this subscale likely overlaps with OCD symptoms.

An additional finding was that HMPS-other-oriented perfectionism consistently explained significant amounts of variance; it was associated with a lower effect size in every meta-regression analysis it was evaluated in. Although it has been stated that HMPS-otheroriented perfectionism is somewhat different from the other perfectionism dimensions (Stoeber \& Otto, 2006), the present findings support the inclusion of HMPS-other-oriented perfectionism in the broad dimension perfectionistic strivings, as proposed by (Bieling, Israeli, et al., 2004). However, in the evaluation of weighted average effect sizes HMPSother-oriented perfectionism was not significantly correlated with most of the outcomes except for global eating pathology, dietary restraint, and deliberate self-harm. Future research is needed to investigate the relationship of HMPS-other-oriented perfectionism to psychopathology in more detail and to determine whether it can be subsumed with the remaining dimensions or not.

In summary, the two main dimensions of perfectionism (perfectionistic concerns and perfectionistic strivings) consistently explained significant amounts of variance whereas the subscales from different inventories could not explain variance in most of the meta-regression models other than those mentioned above. Thus, the formation of the two main dimensions as involving various subscales is supported; evaluating the single subscales separately can however give insight into specific patterns of perfectionism in certain types of psychopathology. 


\section{Implications}

This is the first meta-analysis of tests of effects between perfectionism and psychopathology. Findings indicate substantial overlap in the two perfectionism dimensions in the context of various psychological disorders, their symptoms, and outcomes related to psychopathology. The application of meta-analytic methods offered the chance to resolve inconsistencies observed in the literature attributable to methodological artefacts.

In terms of theoretical implications, the findings suggest that perfectionism needs to be considered in the context of a variety of disorders. As outlined, a transdiagnostic process is a one which is involved in the maintenance of multiple psychological disorders (Harvey et al., 2004). Egan et al. (2011) argued thatperfectionism is a transdiagnostic process because it is (i) elevated across eating disorders, anxiety disorders, OCD and depression compared to healthy controls; (ii) a risk and maintaining factor across disorders, and (iii) associated with co-occurring psychological disorders. Consistent with this is a study of 345 people with cooccurring anxiety and mood disorders that found the number of diagnoses was positively correlated with perfectionism and that perfectionism predicted higher co-occurrence of disorders even after controlling for symptoms (Bieling, Summerfeldt, Israeli, \& Antony, 2004). Bieling and colleagues (Bieling, Summerfeldt, et al., 2004) concluded that treating perfectionism will be more beneficial in patients with co-occurring disorders than disorder specific treatments and may result in symptom reduction across multiple disorders. The current meta-analytic findings support the assertion based on the previous narrative review of Egan et al. (2011) that perfectionism is a shared etiological factor in OCD, anxiety disorders, depression and eating disorders. Future research should examine the reason for this, for example by considering factors including whether it is the relationship of perfectionism to comorbidity or shared symptoms between disorders, and how perfectionism is an etiological factor across disorders. There are many potential etiological factors for perfectionism which may be relevant and some have been described elsewhere such as parental and cognitive 
factors (e.g., Maloney, Egan, Kane, \& Rees, 2014), however others such as an overall deficit in ones sense of self or core low self-esteem may also be useful to investigate further in order to inform the theoretical understanding of how perfectionism is a transdiagnostic process.

The clinical implications of the study are speculative based on our data. In summary, our findings suggest that although perfectionism is not specific to a particular form of psychopathology, decreasing perfectionism through CBT for perfectionism could be beneficial in the context of a variety of psychopathological outcomes given there is metaanalytic evidence for efficacy in a range of symptoms across disorders (Lloyd et al., 2015). Further, evaluating specific patterns of perfectionism and comparing scores on subscales of multidimensional measures could help develop a more detailed picture of specific cognitions and inform treatment in a more targeted manner than through concentrating solely on the broad perfectionistic concerns and perfectionistic strivings dimensions. Current treatment approaches in CBT for perfectionism (Egan, Wade, et al., 2014) emphasise the reduction of perfectionistic concerns but not perfectionistic strivings. This approach appears to be supported for clients with diagnoses of OCD, anxiety disorders and depression, given the evidence we found for perfectionistic concerns having a stronger relationship with these disorders than perfectionistic strivings which had small or trivial associations. However, given our findings that perfectionistic strivings was strongly associated with eating disorder outcomes along with perfectionistic concerns, it appears that a different approach may be required when targeting clients presenting for perfectionism treatment who meet a diagnosis of an eating disorder. The current treatment emphasis has been on explicitly stating to the client early in therapy that there is nothing wrong with striving for standards in itself (i.e., perfectionistic strivings), but it is the concern over mistakes (i.e., perfectionistic concerns) and basing ones self-worth on striving and achievement that is problematic (Egan, Wade, et al., 2014). Given our findings it would be useful for future research to determine if changing this approach for those presenting with eating disorders would be more effective than the 
current treatment, specifically where it is investigated if modifying treatment in order to reduce perfectionistic strivings results in stronger effects in reduction of eating disorder symptoms. This would also be an interesting line of research to examine regarding eating disorder prevention, where perfectionism has been a recent focus of interest, in order to determine if prevention programs for perfectionism should be modified to explicitly focus on reducing both perfectionistic strivings and perfectionistic concerns.

\section{Strengths and Limitations}

The key strength of the current analysis was the comprehensive literature search and inclusion criteria for studies on perfectionism and the adoption of meta-analytic techniques to estimate bias-corrected tests of relations among perfectionism and psychopathology outcomes across the extant literature. A further advantage is the adoption of state-of-the-art meta-analytic techniques using robust variance estimation (Hedges et al., 2010) which allowed including multiple effect sizes from single studies while controlling for data dependencies. Furthermore, through the simultaneous evaluation of a variety of psychological disorders, symptoms, and outcomes related to psychopathology this study has provided the first meta-analysis to enable a detailed understanding of the role of perfectionism in a range of psychopathology.

A limitation was the various measures used for perfectionism and outcomes, thus assessing the relationship was more difficult than if the same measures had been used. This is likely to have introduced further methodological variance. Moreover, the variety of investigated outcomes led to the need to categorise them into broader groups. For example, anxiety disorders had to be subsumed into one category instead of keeping the different anxiety disorders separate. Thus, possible differences between the anxiety disorders have not been addressed in this review. A similar issue appeared for OCD and eating disorders: we were unable to draw conclusions on specific subtypes of OCD or specific eating disorders like anorexia and bulimia nervosa in the meta-regression analyses due to too few studies in 
the current sample conducting analysis of the respective subtypes separately. In addition, the clinical meaning of the present findings is weakened by the fact that more studies on nonclinical than on clinical samples were included. This fact may also serve to help explaining the differences found in the meta-regression models restricted to clinical vs. non-clinical samples: The tendency of a lower influence of perfectionistic strivings in clinical samples was not significant, possibly due to a lower number of studies in clinical samples. Furthermore, due to the same reason some subscales could not be evaluated in clinical samples at all.

Further, although we examined gender as a moderator, we were unable to draw valid conclusions as the majority of the samples (74\%) were female. In particular, we were unable to examine perfectionism in young men due to the low percentage of male samples in the studies collected. Future research investigating relations between perfectionism and outcomes in men is advocated in order to develop an evidence base to allow for better tests of gender differences in this literature.

Another limitation was that although we argued that identifying which perfectionism measure exhibits the strongest relationship with psychopathology is important as it may inform clinical interventions, a problem with this is that it is possible that the correlation between perfectionism measures and symptoms may be due to shared method variance (i.e., both are single informant and self-report) or due to some measures potentially having overlap with the specific symptoms of the disorder. This is particularly the case when considering the strong association between doubts about actions and OCD, as outlined previously this perfectionism subscale has been criticised as being highly overlapping with OCD symptoms (Shafran \& Mansell, 2001). While we do not believe this is the case for all measures of perfectionism, as some do not overlap with psychopathology symptoms, it is possible that some of our results such as those in OCD may have inflated associations due to this overlap and this is a limitation to be acknowledged. 
A further limitation of this study is that only 18 of the 284 studies that were included were longitudinal, highlighting that a problem with the current literature on perfectionism, as is the case with most psychological research, that the data for the field, on the whole, does not adopt strong, longitudinal designs. Correlational designs hold back the field of research and limit the inferences that researchers and practitioners can make with respect to understanding theory and interventions. While one recent meta-analysis exists looking at longitudinal studies of depression in perfectionism (Smith et al., 2016), given we followed the guideline that ten primary studies are needed to evaluate moderator variables (Dalton \& Dalton, 2008), we were unable to conduct separate analyses predicting longitudinal development for the single clinical disorders or symptom categories which is a limitation.

Consequently, no conclusion as to whether perfectionism is as a risk or maintenance factor for psychopathology can be made due to the fact that insufficient longitudinal studies about the relationship between psychological disorders and perfectionism exist. Therefore it is not possible to say whether perfectionism leads to the onset of particular disorders or symptom. Thus, conclusions on whether reducing perfectionism would reduce symptoms of a disorder are not possible. We issue a call to the field to reduce the use of cross-sectional research and instead encourage future researchers to conduct longitudinal research, most importantly, longitudinal, cross-lagged panel designs and experimental research looking to change perfectionism dimensions and observe the effects on psychopathology outcomes.

Concerning the analysis of single subscales of FMPS and HMPS, the mostly nonsignificant findings may be due to the fact that there was more power to detect differences with the dimensional approach than the subscale approach because there were more studies for the former. Another limitation was that we only included published or in press studies in order to minimize potential duplication of findings. However, this approach may also mean that we may omitted studies that have been completed but not yet published or still under review. However, it is important to note that we inspected the funnel plot of study precision 
against effect size and applied Egger et al.'s (1997) regression techniques to identify potential small study or publication bias in the current set of studies.

Finally, another limitation is that while we included a measure of self-criticism (DASSC; Weissman \& Beck, 1978) which has been used in numerous studies examining the link between perfectionism and psychopathology (e.g.; Dunkley, Sanislow, et al., 2006; Dunkley et al., 2009) there are other scales which we did not include such as the Depressive Experiences Questionnaire (DEQ; Blatt, D'Aflitti, \& Quinlan, 1979) and the SociotropyAutonomy Scale (SAS; Beck, Epstein, Harrison, \& Emery, 1983) which have been found in some factor analytic studies to load on to a self-critical perfectionism factor (e.g., Dunkley, Blankstein, Zuroff, Lecce, \& Hui, 2006). These other scales were not included due to our focus specifically on perfectionistic strivings and perfectionistic concerns and measures specifically designed to assess perfectionism (e.g. MPS; Frost et al., 1990; Hewitt \& Flett, 1991b), and our inclusion criteria reflect this. An analysis of all measures of self-criticism was beyond the scope of the current analysis, however we look to future research to expand current findings to include other measures such as the DEQ and SAS which share conceptual overlap with perfectionism.

\section{Future Research}

As the current meta-analysis was conducted on studies that mostly used non-clinical samples and thus concentrated on symptoms of disorders, it would be important to again investigate the relation of perfectionism to clinical diagnoses in addition to symptoms when sufficient data become available. Thus, although many primary studies on the role of perfectionism in the context of specific disorders exist, further studies are needed. This would allow more meaningful conclusions on the clinical role of perfectionism. It would be worthwhile to establish the role of perfectionism as a risk or maintenance factor in the context of different disorders in order to propose suggestions for prevention and treatment of a variety of disorders. This could be done by further examination of longitudinal associations 
between perfectionism and clinical outcomes using meta-analytic methods. As outlined, a problem in the research area is the preponderance of cross-sectional studies relative to longitudinal studies, and future researchers are encouraged to consider longitudinal designs in order to provide more robust evidence of the relationships between perfectionism and psychopathology.

The present review did not evaluate moderators of the relationship between perfectionism and psychopathology such as duration of illness, treatment seeking and methodology; further, as stated before, age and gender could not be evaluated sufficiently due to low proportions of male participants and similar age groups in primary studies. More primary studies giving information and statistical variance on these characteristics as well as studies with wider age spans and the inclusion of male participants are needed to allow insight by way of meta-analysis.

Another direction for future research would be to consider whether being high on both perfectionistic concerns and perfectionistic strivings at the same time confers a "dual" vulnerability in the sense of being elevated on both dimensions of perfectionism. This may explain why perfectionistic strivings is more strongly linked to psychopathology in clinical samples, largely relating to eating disorder outcomes, that is, individuals with clinical disorders may show elevations in both perfectionism dimensions, and this differentiates them from control samples.

Further meta-analytic research would be useful to evaluate the efficacy of existing interventions for perfectionism. While there has been one systematic review (Lloyd et al., 2015) which examined CBT for perfectionism and found large pooled effect sizes for reductions in perfectionism (FMPS-personal standards, concern over mistakes, HMPS-selforiented perfectionism), and medium pooled effect size reductions for anxiety and depression, only eight studies were included, and further RCTs that have now been published (e.g., Egan, Wade, et al., 2014; Handley et al., 2014) which would be useful to include in an 
updated meta-analysis on treatment efficacy. There are psychological disorders that have just started to gain attention in the context of perfectionism and could not be included in this review due to low numbers of primary studies, for instance, obsessive-compulsive personality disorder, posttraumatic stress disorder, and body-dysmorphic disorder. Extending research on these would help to gain a better understanding of the role of perfectionism in various outcomes. The scope of the present research was not wide enough to evaluate findings specific to particular populations, such as athletes, or perfectionism in other domains, such as dyadic perfectionism, thus they could be evaluated in future meta-analyses. Synthesis of such research will lead to far reaching conclusions for distinct areas, thus shedding further light on the role of perfectionism and the need for effective prevention and intervention. 


\section{Acknowledgements}

The authors wish to thank Rachael Glassey for providing assistance with the original literature search. Thanks to Prof. Thomas Ehring for his helpful comments on a previous version of this paper. 


\section{References}

Antony, M. M., Purdon, C. L., Huta, V., \& Swinson, R. P. (1998). Dimensions of perfectionism across the anxiety disorders. Behaviour Research and Therapy, 36(12), 1143-1154. doi: 10.1016/S0005-7967\%2898\%2900083-7

Bardone-Cone, A. M., Abramson, L. Y., Vohs, K. D., Heatherton, T. F., \& Joiner, T. E. (2006). Predicting bulimic symptoms: An interactive model of self-efficacy, perfectionism, and perceived weight status. Behaviour Research and Therapy, 44(1), 27-42. doi: 10.1016/j.brat.2004.09.009

Bardone-Cone, A. M., Wonderlich, S. A., Frost, R. O., Bulik, C. M., Mitchell, J. E., Uppala, S., \& Simonich, H. (2007). Perfectionism and eating disorders: Current status and future directions. Clinical Psychology Review, 27(3), 384-405.

Beck, A. T., Epstein, N., Harrison, R. P., \& Emery, G. (1983). Development of the Sociotropy-Autonomy Scale: A measure of personality factors in psychopathology. Unpublished manuscript, University of Pennsylvania, Philadelphia,USA.

Békés, V., Dunkley, D. M., Taylor, G., Zuroff, D. C., Lewkowski, M., Foley, J. E., \& Westreich, R. (2015). Chronic stress and attenuated improvement in depression over 1 year: The moderating role of perfectionism. . Behavior Therapy, 46(4), 478-492.

Bieling, P. J., Israeli, A. L., \& Antony, M. M. (2004). Is perfectionism good, bad, or both? Examining models of the perfectionism construct. Personality and Individual Differences, 36(6), 1373-1385. doi: 10.1016/S0191-8869\%2803\%2900235-6

Bieling, P. J., Summerfeldt, L. J., Israeli, A. L., \& Antony, M. M. (2004). Perfectionism as an explanatory construct in comorbidity of axis I disorders. Journal of Psychopathology and Behavioral Assessment, 26(3), 193-201.

Black, K. (2009). Business statistics: Contemporary decision making (6th ed.): Wiley.

Blatt, S. J., D'Aflitti, J. P., \& Quinlan, D. M. (1979). Depressive Experiences Questionnaire. Unpublished manuscript. New Haven, CT. 
Borenstein, M., Hedges, L., Higgins, J., \& Rothstein, H. (2005). Comprehensive metaanalysis version 2. Englewood, NJ: Biostat.

Borenstein, M., Hedges, L. V., Higgins, J. P., \& Rothstein, H. R. (2009). Introduction to meta-analysis. West Sussex, UK: John Wiley \& Sons, Ltd.

Brannan, M. E., \& Petrie, T. A. (2008). Moderators of the body dissatisfaction-eating disorder symptomatology relationship: replication and extension. Journal of Counseling Psychology, 55(2), 263-275.

Buhlmann, U., Etcoff, N. L., \& Wilhelm, S. (2008). Facial attractiveness ratings and perfectionism in body dysmorphic disorder and obsessive-compulsive disorder. Journal of Anxiety Disorders, 22(3), 540-547. doi: 10.1016/j.janxdis.2007.05.004

Cockell, S. J., Hewitt, P. L., Seal, B., Sherry, S., Goldner, E. M., Flett, G. L., \& Remick, R. A. (2002). Trait and self-presentational dimensions of perfectionism among women with anorexia nervosa. Cognitive Therapy and Research, 26(6), 745-758. doi: 10.1023/A:1021237416366

Cohen, J. (1988). Statistical power analysis for the behavioral sciences (2nd ed.). Hillsdale, NJ: Lawrence Earlbaum Associates.

Dalton, D. R., \& Dalton, C. M. (2008). Meta-Analyses Some Very Good Steps Toward a Bit Longer Journey. Organizational Research Methods, 11(1), 127-147.

Dunkley, D. M., Blankstein, K. R., Zuroff, D. C., Lecce, S., \& Hui, D. (2006). Self-critical and personal standards factors of perfectionism located within the five-factor model of personality. . Personality and Individual Differences, 40(3), 409-420.

Dunkley, D. M., Sanislow, C. A., Grilo, C. M., \& McGlashan, T. H. (2006). Perfectionism and depressive symptoms 3 years later: Negative social interactions, avoidant coping, and perceived social support as mediators. Comprehensive Psychiatry, 47(2), 106115. doi: 10.1016/j.comppsych.2005.06.003 
Dunkley, D. M., Sanislow, C. A., Grilo, C. M., \& McGlashan, T. H. (2009). Self-criticism versus neuroticism in predicting depression and psychosocial impairment for 4 years in a clinical sample. . Comprehensive Psychiatry, 50(4), 335-346.

Egan, S. J., Hattaway, M., \& Kane, R. T. (2014). The relationship between perfectionism and rumination in post traumatic stress disorder. Behavioural and cognitive psychotherapy, 42(2), 211-223.

Egan, S. J., Wade, T., Shafran, R., \& Antony, M. M. (2014). Cognitive-behavioral treatement for perfectionism. New York: Guilford Publications.

Egan, S. J., Wade, T. D., \& Shafran, R. (2011). Perfectionism as a transdiagnostic process: a clinical review. Clinical Psychology Review, 31(2), 203-212. doi: 10.1016/j.cpr.2010.04.009

Egger, M., Smith, G. D., Schneider, M., \& Minder, C. (1997). Bias in meta-analysis detected by a simple, graphical test. Bmj, 315(7109), 629-634.

Enns, M. W., Cox, B. J., \& Borger, S. C. (2001). Correlates of analogue and clinical depression: A further test of the phenomenological continuity hypothesis. Journal of Affective Disorders, 66(2-3), 175-183. doi: 10.1016/S0165-0327\%2800\%2900305-0

Enns, M. W., Cox, B. J., Sareen, J., \& Freeman, P. (2001). Adaptive and maladaptive perfectionism in medical students: A longitudinal investigation. Medical Education, 35(11), 1034-1042. doi: 10.1046/j.1365-2923.2001.01044.x

Fairburn, C. G., Cooper, Z., \& Shafran, R. (2003a). Cognitive behaviour therapy for eating disorders: A "transdiagnostic" theory and treatment. Behaviour Research and Therapy, 41(5), 509-528.

Fairburn, C. G., Cooper, Z., \& Shafran, R. (2003b). Cognitive behaviour therapy for eating disorders: A "transdiagnostic" theory and treatment. Behaviour Research and Therapy, 41(5), 509-528. 
Flett, G., Hewitt, P., Boucher, D., Davidson, L., \& Munro, Y. (1997). The child-adolescent perfectionism scale: Development, validation, and association with adjustment. Unpublished manuscript.

Frost, R. O., Heimberg, R. G., Holt, C. S., \& Mattia, J. I. (1993). A comparison of two measures of perfectionism. Personality and Individual Differences, 14(1), 119-126. doi: 10.1016/0191-8869\%2893\%2990181-2

Frost, R. O., Marten, P., Lahart, C., \& Rosenblate, R. (1990). The dimensions of perfectionism. Cognitive therapy and research, 14(5), 449-468.

Frost, R. O., \& Steketee, G. (1997). Perfectionism in obsessive-compulsive disorder patients. Behaviour Research and Therapy, 35(4), 291-296. doi: http://dx.doi.org/10.1016/S0005-7967\%2896\%2900108-8

Garner, D. M., Olmstead, M. P., \& Polivy, J. (1983). Development and validation of a multidimensional eating disorder inventory for anorexia nervosa and bulimia. International journal of eating disorders, 2(2), 15-34.

Gnilka, P. B., Ashby, J. S., \& Noble, C. M. (2012). Multidimensional perfectionism and anxiety: Differences among individuals with perfectionism and tests of a copingmediation model. Journal of Counseling \& Development, 90(4), 427-436. doi: 10.1002/j.1556-6676.2012.00054.x

Graham, A. R., Sherry, S. B., Stewart, S. H., Sherry, D. L., McGrath, D. S., Fossum, K. M., \& Allen, S. L. (2010). The existential model of perfectionism and depressive symptoms: A short-term, four-wave longitudinal study. Journal of Counseling Psychology, 57(4), 423-438.

Hagger, M. S., \& Chatzisarantis, N. L. (2014). It is premature to regard the ego-depletion effect as "Too Incredible". Frontiers in psychology, 5.

Halmi, K. A., Sunday, S. R., Strober, M., Kaplan, A., Woodside, D. B., Fichter, M., . . Kaye, W. H. (2000). Perfectionism in anorexia nervosa: variation by clinical subtype, 
obsessionality, and pathological eating behavior. American Journal of Psychiatry, 157(11), 1799-1805.

Handley, A. K., Egan, S. J., Kane, R. T., \& Rees, C. S. (2014). The relationships between perfectionism, pathological worry and generalised anxiety disorder. BMC psychiatry, 14(1).

Harvey, A., Watkins, E., Mansell, W., \& Shafran, R. (2004). Cognitive behavioural processes across psychological disorders: A transdiagnostic approach to research and treatment. Oxford, UK: Oxford University Press.

Hedges, L. V., \& Olkin, I. (1985). Statistical methods for meta-analysis. New York: Academic Press.

Hedges, L. V., Tipton, E., \& Johnson, M. C. (2010). Robust variance estimation in metaregression with dependent effect size estimates. Research Synthesis Methods, 1(1), $39-65$.

Hewitt, P. L., \& Flett, G. L. (1991a). Dimensions of Perfectionism in Unipolar Depression. Journal of Abnormal Psychology, 100(1), 98-101.

Hewitt, P. L., \& Flett, G. L. (1991b). Perfectionism in the self and social contexts: conceptualization, assessment, and association with psychopathology. Journal of Personality and Social Psychology, 60(3), 456-470.

Hewitt, P. L., Flett, G. L., \& Ediger, E. (1996). Perfectionism and depression: Longitudinal assessment of a specific vulnerability hypothesis. Journal of Abnormal Psychology, 105(2), 276-280. doi: 10.1037/0021-843X.105.2.276

Hodgson, R. J., \& Rachman, S. (1977). Obsessional compulsive complaints. Behaviour Research and Therapy, 15, 389-395.

Huprich, S. K., Porcerelli, J., Keaschuk, R., Binienda, J., \& Engle, B. (2008). Depressive personality disorder, dysthymia, and their relationship to perfectionism. Depression and Anxiety, 25(3), 207-217. doi: 10.1002/da.20290 
IBM Corp. (2012). IBM SPSS Statistics for Windows, Version 21.0. Armonk, NY: IBM Corp.

Iketani, T., Kiriike, N., Stein, M. B., Nagao, K., Nagata, T., Minamikawa, N., . . Fukuhara, H. (2002). Relationship between perfectionism and agoraphobia in patients with panic disorder. Cognitive Behaviour Therapy, 31(3), 119-128. doi:

$10.1080 / 165060702320337997$

Juster, H. R., Heimberg, R. G., Frost, R. O., Holt, C. S., Mattia, J. I., \& Faccenda, K. (1996). Social phobia and perfectionism. Personality and Individual Differences, 21(3), 403410. doi: 10.1016/0191-8869(96)00075-x

Lilenfeld, L., Stein, D., Bulik, C., Strober, M., Plotnicov, K., Pollice, C., . . Kaye, W. H. (2000). Personality traits among current eating disordered, recovered and never ill first-degree female relatives of bulimic and control women. Psychological Medicine, 30(6), 1399-1410. doi: 10.1017/S0033291799002792

Lloyd, S., Schmidt, U., Khondoker, M., \& Tchanturia, K. (2015). Can psychological interventions reduce perfectionism? A systematic review and meta-analysis. Behavioural and cognitive psychotherapy, 43(6), 705-731.

Lombardo, C., Mallia, L., Battagliese, G., Grano, C., \& Violani, C. (2013). Perfectionism mediates the relationship between insomnia and depressive symptoms. Sleep and Biological Rhythms, 11(2), 90-98.

Maloney, G. K., Egan, S. J., Kane, R. T., \& Rees, C. S. (2014). An etiological model of perfectionism. . PloS one, 9(5), e94757.

Miller-Day, M., \& Marks, J. D. (2006). Perceptions of parental communication orientation, perfectionism, and disordered eating behaviors of sons and daughters. Health Communication, 19(2), 153-163. doi: 10.1207/s15327027hc1902_7 
Moor, S., Vartanian, L. R., Touyz, S. W., \& Beumont, P. (2004). Psychopathology of EDNOS patients: To whom do they compare? Clinical Psychologist, 8(2), 70-75. doi: $10.1080 / 1328420041233130436$

Norman, R. M. G., Davies, F., Nicholson, I. R., Cortese, L., \& Malla, A. K. (1998). The relationship of two aspects of perfectionism with symptoms in a psychiatric outpatient population. Journal of Social and Clinical Psychology, 17(1), 50-68. doi: 10.1521/jscp.1998.17.1.50

Obsessive Compulsive Cognitions Working Group. (2001). Development and initial validation of the Obsessive Beliefs Questionnaire and the Interpretation of Intrusions Inventory. Behaviour Research and Therapy.

Rhéaume, J., Freeston, M. H., Dugas, M. J., Letarte, H., \& Ladouceur, R. (1995). Perfectionism, responsibility and obsessive-compulsive symptoms. Behaviour Research and Therapy, 33(7), 785-794.

Rhéaume, J., Freeston, M. H., Ladouceur, R., Bouchard, C., Gallant, L., Talbot, F., \& Vallières, A. (2000). Functional and dysfunctional perfectionists: are they different on compulsive-like behaviors? Behaviour Research and Therapy, 38(2), 119-128.

Rice, K. G., Kubal, A. E., \& Preusser, K. J. (2004). Perfectionism and children's self-concept: Further validation of the Adaptive/Maladaptive Perfectionism Scale. Psychology in the Schools, 41(3), 279-290. doi: 10.1002/pits.10160

Rodriguez-Seijas, C., Eaton, N. R., \& Krueger, R. F. . (2015). How transdiagnostic factors of personality and psychopathology can inform clinical assessment and intervention. Journal of Personality Assessment, 97(5), 425-435.

Rosenthal, R. (1979). The file drawer problem and tolerance for null results. Psychological Bulletin, 86(3), 638. 
Saboonchi, F., Lundh, L.-G., \& Ost, L.-G. (1999). Perfectionism and self-consciousness in social phobia and panic disorder with agoraphobia. Behaviour Research and Therapy, 37(9), 799-808. doi: 10.1016/S0005-7967\%2898\%2900183-1

Sassaroli, S., Lauro, L. J. R., Ruggiero, G. M., Mauri, M. C., Vinai, P., \& Frost, R. (2008). Perfectionism in depression, obsessive-compulsive disorder and eating disorders. Behaviour Research \& Therapy, 46(6), 757-765. doi: 10.1016/j.brat.2008.02.007

Schmidt, U., \& Treasure, J. (2006). Anorexia nervosa: Valued and visible. A cognitiveinterpersonal maintenance model and its implications for research and practice. British Journal of Clinical Psychology, 45(3), 343-366.

Shafran, R., Cooper, Z., \& Fairburn, C. G. (2003). "Clinical perfectionism" is not "multidimensional perfectionism": a reply to Hewitt, Flett, Besser, Sherry \& McGee. Behaviour Research and Therapy, 41, 1217-1220.

Shafran, R., \& Mansell, W. (2001). Perfectionism and psychopathology: A review of research and treatment. Clinical Psychology Review, 21(6), 879-906. doi: 10.1016/S0272$7358 \% 2800 \% 2900072-6$

Slaney, R. B., Rice, K. G., Mobley, M., Trippi, J., \& Ashby, J. S. (2001). The Revised Almost Perfect Scale. Measurement and Evaluation in Counseling and Development.

Smith, M. M., Sherry, S. B., Rnic, K., Saklofske, D. H., Enns, M., \& Gralnick, T. (2016). Are Perfectionism Dimensions Vulnerability Factors for Depressive Symptoms After Controlling for Neuroticism? A Meta-analysis of 10 Longitudinal Studies. . European Journal of Personality, 30(2), 201-212.

Stoeber, J., \& Otto, K. (2006). Positive conceptions of perfectionism: Approaches, evidence, challenges. Personality and social psychology review, 10(4), 295-319.

Tanner-Smith, E. E., Wilson, S. J., \& Lipsey, M. W. (2013). The comparative effectiveness of outpatient treatment for adolescent substance abuse: A meta-analysis. Journal of substance abuse treatment, 44(2), 145-158. 
Terry-Short, L., Owens, R. G., Slade, P., \& Dewey, M. (1995). Positive and negative perfectionism. Personality and Individual Differences, 18(5), 663-668.

Viswesvaran, C., \& Ones, D. S. (1995). Theory testing: Combining psychometric metaanalysis and structural equations modeling. Personnel Psychology, 48(4), 865-885.

Weissman, A. N., \& Beck, A. T. (1978). Development and validation of the Dysfunctional Attitude Scale: A preliminary investigation.

Welch, E., Miller, J. L., Ghaderi, A., \& Vaillancourt, T. (2009). Does perfectionism mediate or moderate the relation between body dissatisfaction and disordered eating attitudes and behaviors? Eating Behaviors, 10(3), 168-175. doi: 10.1016/j.eatbeh.2009.05.002 
Table 1

Scales Measuring Perfectionism along with the Classification of their Subscales into the Two Major Dimensions of Perfectionism

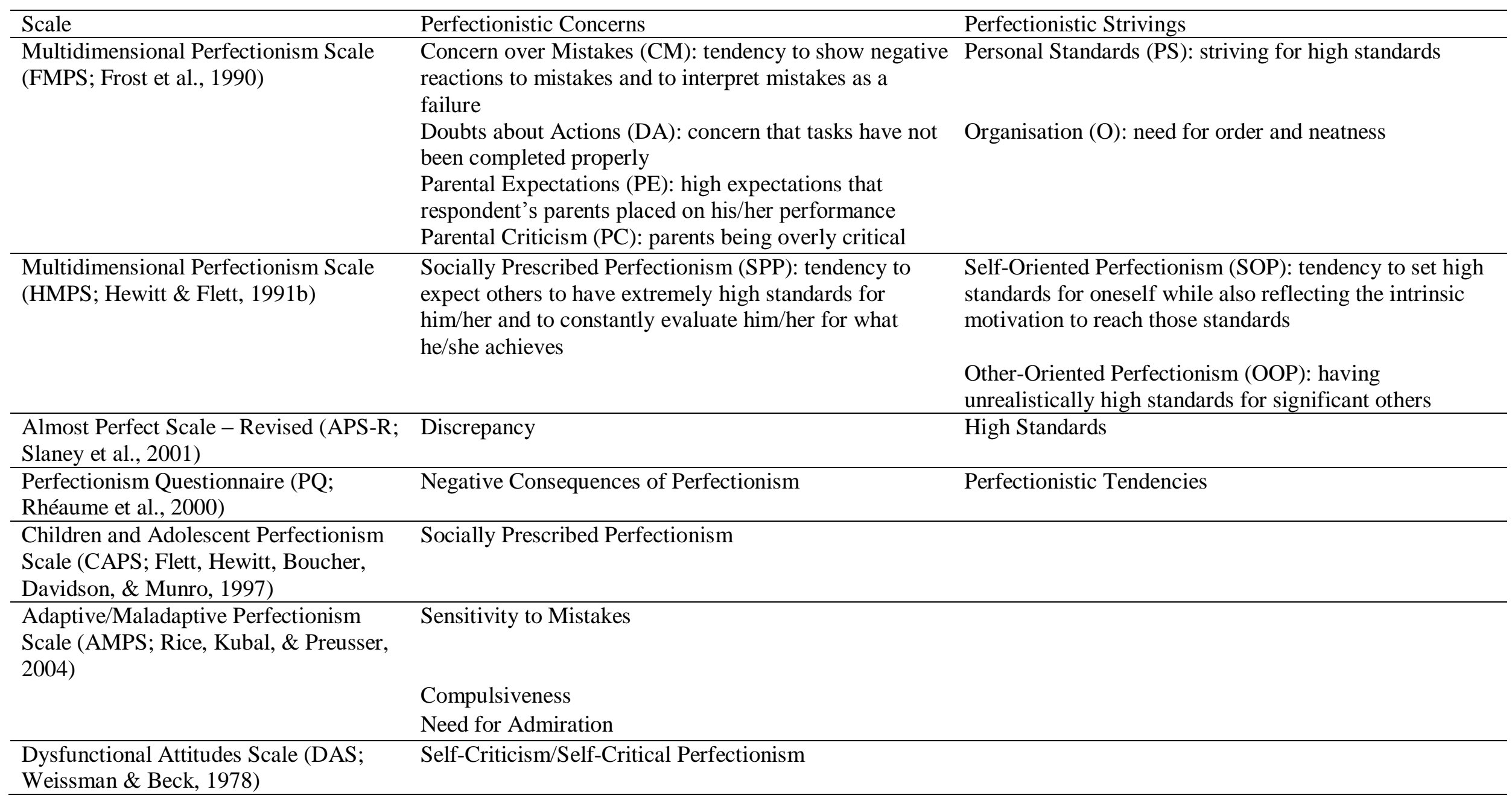


Clinical Perfectionism Questionnaire

Perfectionism

(CPQ; Fairburn, Cooper, \& Shafran,

2003b)

Positive and Negative Perfectionism

Negative Perfectionism

Scale (PANPS; Terry-Short, Owens,

Slade, \& Dewey, 1995)

Obsessive Beliefs Questionnaire

(Obsessive Compulsive Cognitions

Working Group [OCCWG], 2001)

Perfectionism subscale of the Eating

Disorder Inventory (EDI-P;Garner,

Perfectionism

Olmstead, \& Polivy, 1983) 


\section{Table 2}

Weighted Averaged Zero-Order (r) and Unique ( $\beta$ ) Effect Sizes with Confidence Intervals for Relations between the Two Main Dimensions of Perfectionism and Psychological Disorders, Symptoms, and Psychopathological Outcomes

\begin{tabular}{|c|c|c|c|c|c|c|c|c|c|c|c|c|c|c|c|c|c|}
\hline \multirow[t]{3}{*}{ Outcome } & & \multirow[t]{3}{*}{$k$} & \multirow[t]{3}{*}{$n$} & \multicolumn{6}{|c|}{ Perfectionistic concerns } & \multirow[t]{3}{*}{$k$} & \multirow[t]{3}{*}{$n$} & \multicolumn{6}{|c|}{ Perfectionistic strivings } \\
\hline & & & & \multirow[t]{2}{*}{$r$} & \multicolumn{2}{|c|}{$95 \%$ CI of $r$} & \multirow[t]{2}{*}{$\beta$} & \multicolumn{2}{|c|}{$95 \%$ CI of $\beta$} & & & \multirow[t]{2}{*}{$r$} & \multicolumn{2}{|c|}{$95 \% \mathrm{CI}$ of $r$} & \multirow{2}{*}{$\beta$} & \multicolumn{2}{|c|}{$95 \% \mathrm{CI}$ of $\beta$} \\
\hline & & & & & $L L$ & $U L$ & & $L L$ & $U L$ & & & & $L L$ & $U L$ & & $L L$ & $U L$ \\
\hline \multirow[t]{5}{*}{$\begin{array}{l}\text { Psychological } \\
\text { disorders }\end{array}$} & Depression & 9 & 12 & $.40^{* * * *}$ & .29 & .50 & $.40^{* * *}$ & .32 & .48 & 7 & 8 & $.18^{*}$ & .04 & .32 & .01 & -.08 & .09 \\
\hline & Anxiety disorders & 20 & 49 & $.30^{* * *}$ & .24 & .36 & $.33^{* * *}$ & .29 & .37 & 16 & 29 & $.07^{* *}$ & .01 & .12 & $-.08^{* * * *}$ & -.12 & -.03 \\
\hline & OCD & 14 & 32 & $.35^{* * *}$ & .24 & .45 & $.37^{* * *}$ & .32 & .43 & 10 & 15 & $.11^{* *}$ & .04 & .18 & $-.06^{*}$ & -.11 & -.01 \\
\hline & Anorexia nervosa & 5 & 8 & .81 & -.20 & .99 & $.70^{* * *}$ & .65 & .75 & 4 & 4 & $.56^{* *}$ & .23 & .78 & $.25^{* * *}$ & .21 & .30 \\
\hline & Bulimia nervosa & 5 & 9 & $.45^{* *}$ & .22 & .64 & $.36^{* * *}$ & .22 & .51 & 4 & 4 & $.36^{* * *}$ & .24 & .47 & $.20^{* *}$ & .06 & .35 \\
\hline \multirow[t]{7}{*}{$\begin{array}{l}\text { Symptoms of } \\
\text { disorders }\end{array}$} & $\begin{array}{l}\text { Depressive } \\
\text { symptoms }\end{array}$ & 151 & 256 & $.39^{* * *}$ & .37 & .41 & $.42^{* * *}$ & .41 & .43 & 118 & 162 & $.11^{* * *}$ & .09 & .14 & $-.08^{* * *}$ & -.09 & -.07 \\
\hline & Anxiety & 69 & 149 & $.35^{* * *}$ & .33 & .38 & $.36^{* * *}$ & .34 & .37 & 48 & 104 & $.14^{* * *}$ & .11 & .17 & $-.02^{* 1}$ & -.03 & -.01 \\
\hline & $\begin{array}{l}\text { Social phobia } \\
\text { symptoms }\end{array}$ & 14 & 38 & $.39^{* * *}$ & .31 & .47 & $.46^{* * *}$ & .45 & .47 & 12 & 26 & .05 & -.03 & .13 & $-.15^{* * *}$ & -.16 & -.14 \\
\hline & Worry & 10 & 11 & $.47^{* * *}$ & .42 & .52 & $.44^{* * *}$ & .40 & .49 & 5 & 5 & $.26^{* * *}$ & .11 & .40 & $.07^{* *}$ & .03 & .11 \\
\hline & OCD symptoms & 29 & 79 & $.30^{* * * *}$ & .25 & .35 & $.30^{* * *}$ & .28 & .32 & 15 & 34 & $.14^{* * *}$ & .09 & .20 & .01 & -.01 & .03 \\
\hline & Obsessive beliefs & 12 & 35 & $.54^{* * *}$ & .45 & .61 & $.49^{* * *}$ & .46 & .52 & 1 & 1 & $.33^{* * *}$ & .23 & .43 & $.12^{* * *}$ & .08 & .15 \\
\hline & $\begin{array}{l}\text { Global eating } \\
\text { pathology }\end{array}$ & 19 & 23 & $.27^{* * *}$ & .23 & .31 & $.22^{* * *}$ & .19 & .25 & 18 & 21 & $.21^{* * *}$ & .23 & .31 & $.11^{* * *}$ & .09 & .14 \\
\hline
\end{tabular}




\begin{tabular}{|c|c|c|c|c|c|c|c|c|c|c|c|c|c|c|c|c|c|}
\hline & Binge eating & 10 & 18 & $.30^{* * *}$ & .26 & .35 & $.32^{* * *}$ & .29 & .35 & 8 & 13 & $.10^{* * * *}$ & .05 & .15 & $-.04^{* * *}$ & -.07 & -.01 \\
\hline & Body dissatisfaction & 24 & 35 & $.32^{* * *}$ & .21 & .42 & $.27^{* * * *}$ & .24 & .29 & 20 & 24 & $.24^{* *}$ & .09 & .35 & $.12^{* * *}$ & .10 & .15 \\
\hline & Dietary restraint & 20 & 27 & $.29^{* * *}$ & .21 & .36 & $.21^{* * *}$ & .19 & .24 & 18 & 22 & $.27^{* * *}$ & .21 & .33 & $.18^{* * *}$ & .15 & .20 \\
\hline & Drive for thinness & 6 & 7 & $.22^{*}$ & .01 & .41 & $.14^{* * *}$ & .09 & .20 & 5 & 5 & $.24^{* * *}$ & .17 & .28 & $.18^{* * *}$ & .12 & .24 \\
\hline & $\begin{array}{l}\text { Thin-ideal } \\
\text { internalisation }\end{array}$ & 2 & 2 & $.21^{* * *}$ & .11 & .30 & $.15^{* * * *}$ & .08 & .22 & 2 & 2 & $.20^{* * * *}$ & .13 & .26 & $.13^{* * *}$ & .06 & .21 \\
\hline \multirow[t]{2}{*}{$\begin{array}{l}\text { Outcomes related to } \\
\text { psychopathology }\end{array}$} & Suicidal ideation & 19 & 22 & $.31^{* * *}$ & .26 & .36 & $.34^{* * *}$ & .30 & .38 & 14 & 15 & $.09^{*}$ & .02 & .15 & $-.06^{* *}$ & -.10 & -.02 \\
\hline & $\begin{array}{l}\text { General } \\
\text { psychological } \\
\text { distress }\end{array}$ & 9 & 17 & $.42^{* * *}$ & .32 & .51 & $.42^{* * * *}$ & .39 & .46 & 9 & 12 & $.18^{* * * *}$ & .12 & .23 & -.01 & -.04 & .03 \\
\hline
\end{tabular}

Note. $k=$ number of tests, $n=$ number of effect sizes, $r=$ Weighted averaged zero-order effect size (correlation) for perfectionism dimensionoutcome relation from meta-analysis; $\mathrm{CI}=$ confidence interval, $L L=$ lower limit, $U L=$ upper limit; $\beta=$ Parameter estimate for unique effect of perfectionism dimension on outcome from path analysis. ${ }^{*} p<0.05,{ }^{*} p<0.01$, *** $p<0.001$. 
Table 3

Weighted Mean Effect Sizes and 95\% Confidence Intervals for the Relationship between subscales of FMPS and HMPS and Several Psychological Disorders Depression

Anxiety disorders

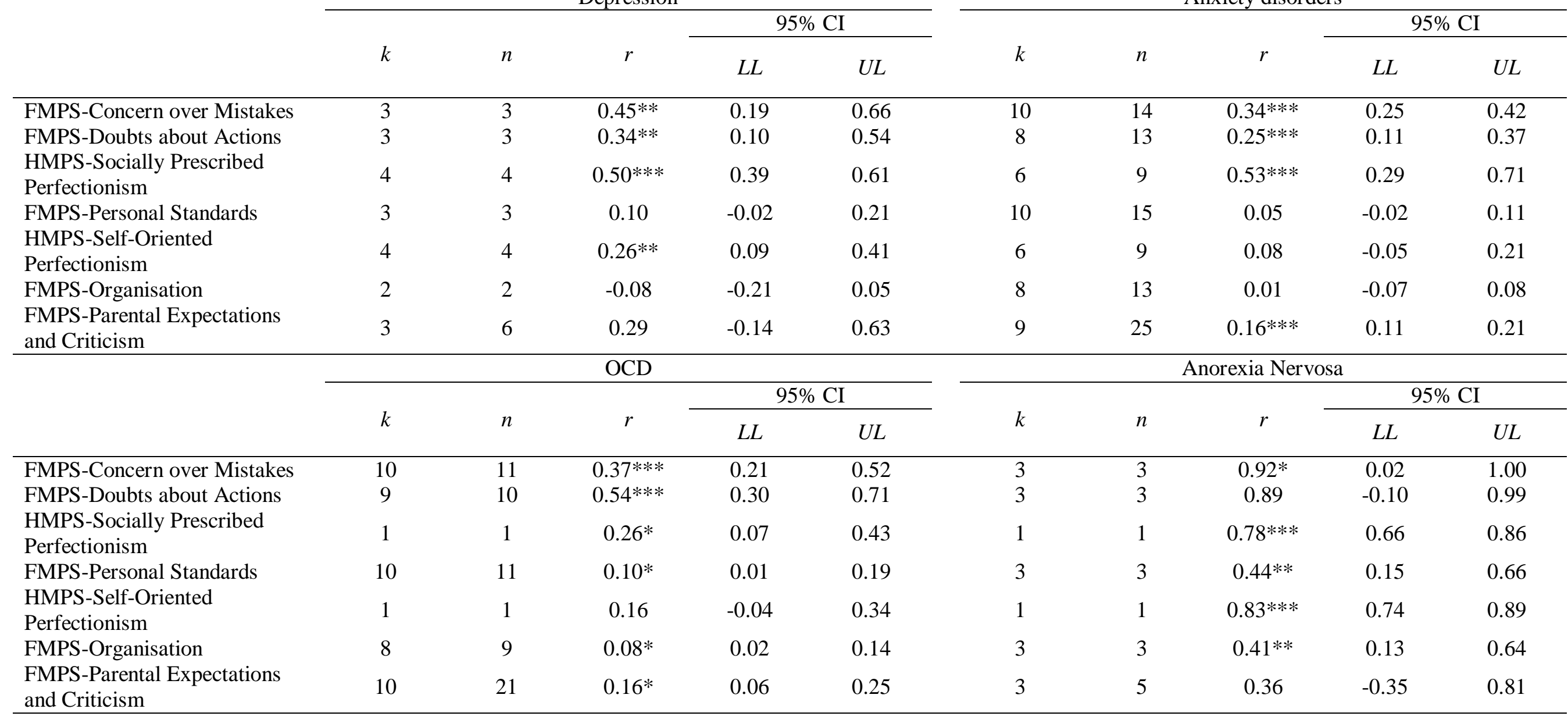

Note. $\mathrm{k}=$ number of tests, $\mathrm{n}=$ number of effect sizes, $C I=$ confidence interval, $\mathrm{LL}=$ lower limit, $\mathrm{UL}=$ upper limit. $* \mathrm{p}<0.05, * * \mathrm{p}<0.01$, $* * * \mathrm{p}<0.001$. 
Table 4

Coefficients and Robust Standard Errors from Nested Meta-Regression Models Predicting Effect Sizes of the Relationship between Perfectionism and Psychopathology by the Subscales of FMPS and HMPS and Sample Characteristics with All Obtained and Relevant Effect Sizes Included

$(\mathrm{k}=322, \mathrm{n}=2,012)$

\begin{tabular}{lccc}
\hline \multirow{2}{*}{ Variable } & \multicolumn{3}{c}{ Model } \\
\cline { 2 - 4 } & \multicolumn{1}{c}{$B$} & $S E$ & $\beta$ \\
\hline Perfectionistic strivings & $-0.21^{* *}$ & 0.06 & -0.14 \\
FMPS-Concern over Mistakes & -0.02 & 0.09 & -0.01 \\
FMPS-Doubts about Actions & 0.15 & 0.11 & 0.05 \\
HMPS-Socially Prescribed Perfectionism & 0.10 & 0.14 & 0.06 \\
FMPS-Personal Standards & 0.13 & 0.08 & 0.05 \\
HMPS-Self-Oriented Perfectionism & -0.03 & 0.13 & -0.02 \\
FMPS-Parental Expectations and Criticism & -0.06 & 0.08 & -0.03 \\
FMPS-Organisation & -0.38 & 0.22 & -0.09 \\
HMPS-Other-Oriented Perfectionism & $-0.35^{* * *}$ & 0.08 & -0.16 \\
Age & $<0.001$ & $<0.001$ & 0.07 \\
Gender (\% female) & $<0.001$ & $<0.001$ & 0.05 \\
Outcome Time (months) & $<0.001$ & $<0.001$ & -0.03 \\
\hline
\end{tabular}

Note. The reference category was Perfectionistic Concerns. Coefficients shown for the between-study effects of variables that varied within and between studies. Age and gender were not provided in $n=35$ cases, that is why the number of effect sizes included here is lower than the overall number of effect sizes. $k=$ number of tests, $n=$ number of effect sizes, $B=$ unstandardized regression coefficient; $S E=$ standard error of $B ; \beta=$ standardized regression coefficient, Outcome time $=$ months between baseline assessment of perfectionism and assessment of outcome.

$* * p<0.01, * * * p<0.001$ 
Table 5

Coefficients and Robust Standard Errors from Nested Meta-Regression Models Predicting Effect Sizes of the Relationship between Perfectionism and Psychopathology by the Subscales of FMPS and HMPS and Sample Characteristics, Restricted to Effect Sizes on Clinical Samples vs. Non-Clinical Samples

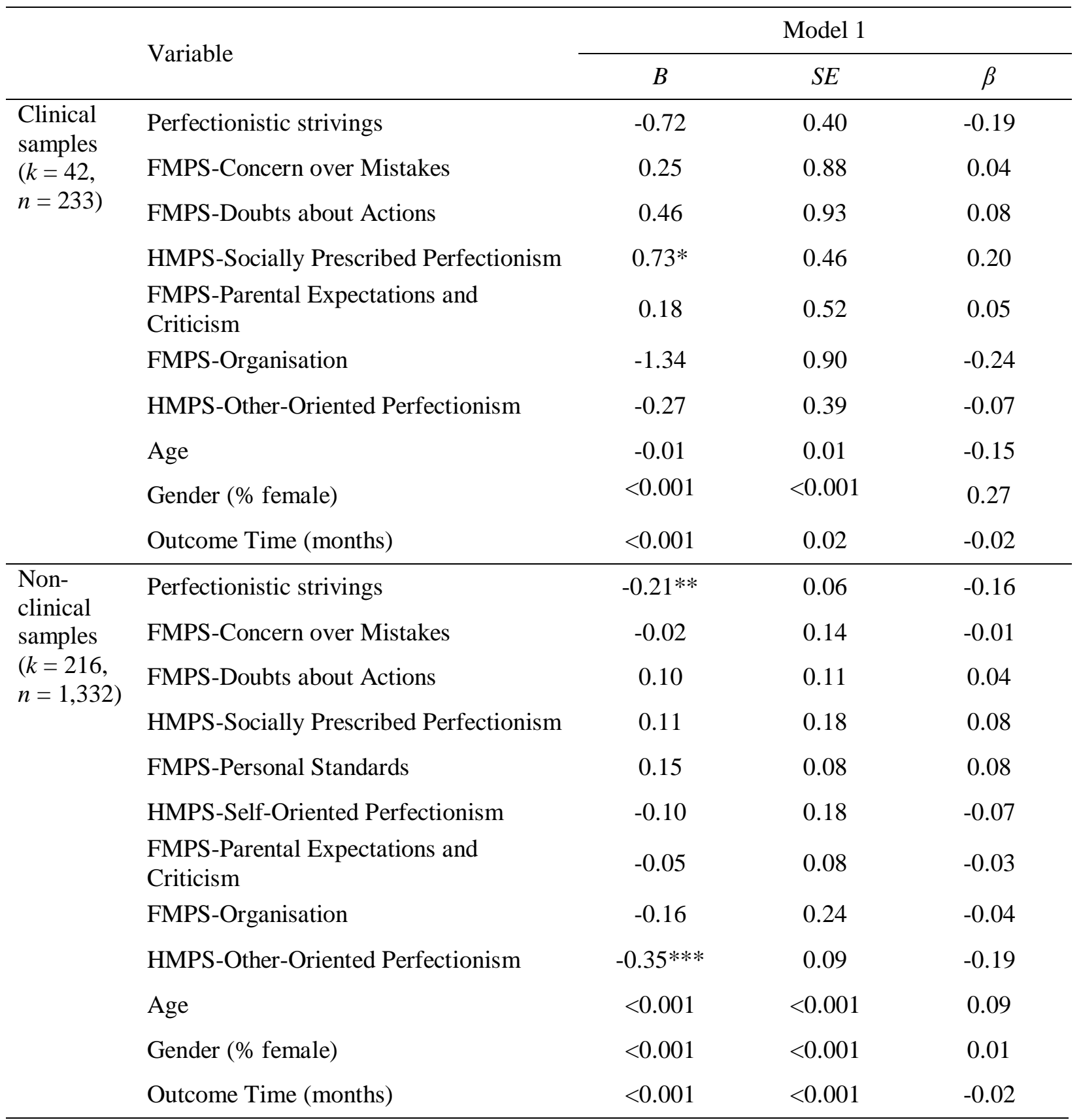

Note. The reference category in both models was perfectionistic concerns. Coefficients shown for the betweenstudy effects of variables that varied within and between studies. $k=$ number of tests, $n=$ number of effect sizes, $B=$ unstandardized regression coefficient, $S E=$ standard error of $B, \beta=$ standardized regression coefficient, Outcome time $=$ months between baseline assessment of perfectionism and assessment of outcome.

$* * p<0.01, * * * p<0.001$. 
Table 6

Coefficients and Robust Standard Errors from Nested Meta-Regression Models Predicting Effect Sizes of the Relationship between Perfectionism and Psychopathology by the Subscales of FMPS and HMPS and Sample Characteristics, Restricted to Effect Sizes on Samples with Anxiety Disorders, OCD, and Eating Disorders, Respectively

\begin{tabular}{|c|c|c|c|c|}
\hline & \multirow{2}{*}{ Variable } & \multicolumn{3}{|c|}{ Model } \\
\hline & & $B$ & $S E$ & $\beta$ \\
\hline \multirow{9}{*}{$\begin{array}{l}\text { Anxiety disorders } \\
(k=20, n=127)\end{array}$} & Perfectionistic strivings & $-0.60 *$ & 0.24 & -0.26 \\
\hline & FMPS-Concern over Mistakes & $1.33 * *$ & 0.32 & 0.37 \\
\hline & FMPS-Doubts about Actions & 0.04 & 0.76 & 0.01 \\
\hline & $\begin{array}{l}\text { HMPS-Socially Prescribed } \\
\text { Perfectionism }\end{array}$ & 0.42 & 0.28 & 0.19 \\
\hline & FMPS-Organisation & $-2.03 *$ & 0.50 & -0.53 \\
\hline & HMPS-Other-Oriented Perfectionism & -0.06 & 0.20 & -0.02 \\
\hline & Age & $<0.001$ & $<0.001$ & 0.04 \\
\hline & Gender ( $\%$ female) & $<0.001$ & $<0.001$ & -0.05 \\
\hline & Outcome Time (months) & 0.02 & 0.01 & 0.17 \\
\hline \multirow{7}{*}{$\begin{array}{l}\text { OCD } \\
(k=12, n=51)\end{array}$} & Perfectionistic strivings & 1.49 & 0.55 & 0.41 \\
\hline & FMPS-Concern over Mistakes & $-10.48 *$ & 3.06 & -1.53 \\
\hline & FMPS-Doubts about Actions & $8.83^{*}$ & 2.05 & 1.55 \\
\hline & HMPS-Self-Oriented Perfectionism & -2.28 & 1.29 & -0.28 \\
\hline & FMPS-Organisation & $-4.77 *$ & 1.57 & -1.02 \\
\hline & Age & $-0.10^{*}$ & 0.03 & -2.46 \\
\hline & Gender ( $\%$ female) & -0.02 & 0.01 & -1.26 \\
\hline \multirow{6}{*}{$\begin{array}{l}\text { Eating disorders } \\
(k=12, n=46)\end{array}$} & Perfectionistic strivings & -1.11 & 3.40 & -0.17 \\
\hline & FMPS-Concern over Mistakes & 0.26 & 2.20 & 0.04 \\
\hline & FMPS-Organisation & -1.13 & 5.05 & -0.12 \\
\hline & Age & 0.04 & 0.09 & 0.25 \\
\hline & Gender ( $\%$ female $)$ & $<0.001$ & 0.01 & 0.08 \\
\hline & Outcome Time (months) & -0.27 & 0.33 & -0.18 \\
\hline
\end{tabular}

Note. The reference category in all models was perfectionistic concerns. Coefficients shown for the betweenstudy effects of variables that varied within and between studies. $k=$ number of tests, $n=$ number of effect sizes, $B=$ unstandardized regression coefficient, $S E=$ standard error of $B, \beta=$ standardized regression coefficient, Outcome time $=$ months between baseline assessment of perfectionism and assessment of outcome.

$* * * p<0.001$. 


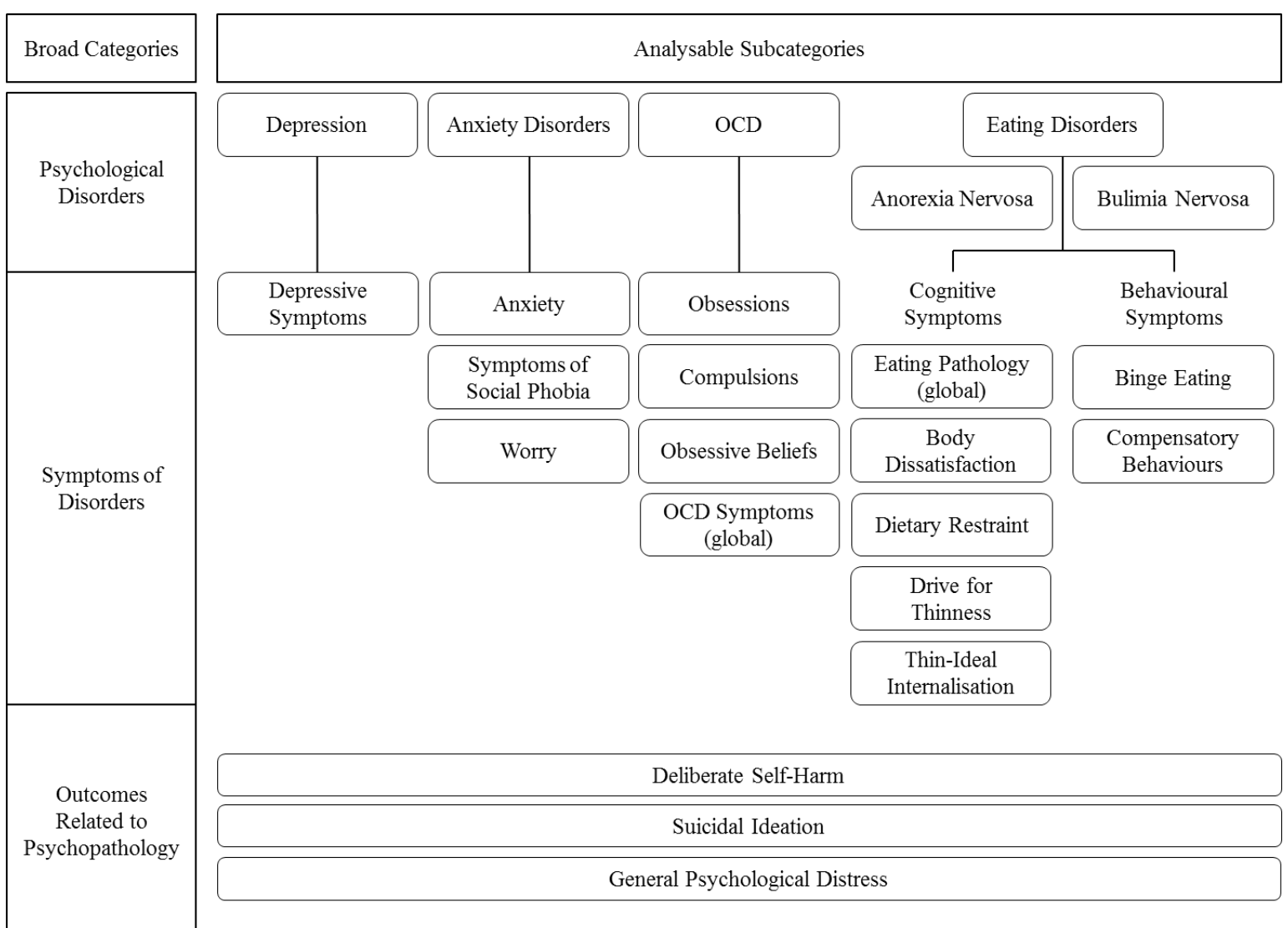

Figure 1. Categories and subcategories of outcomes that were analysed in the meta-analysis. 
Identified in systematic search $(n=2,497)$

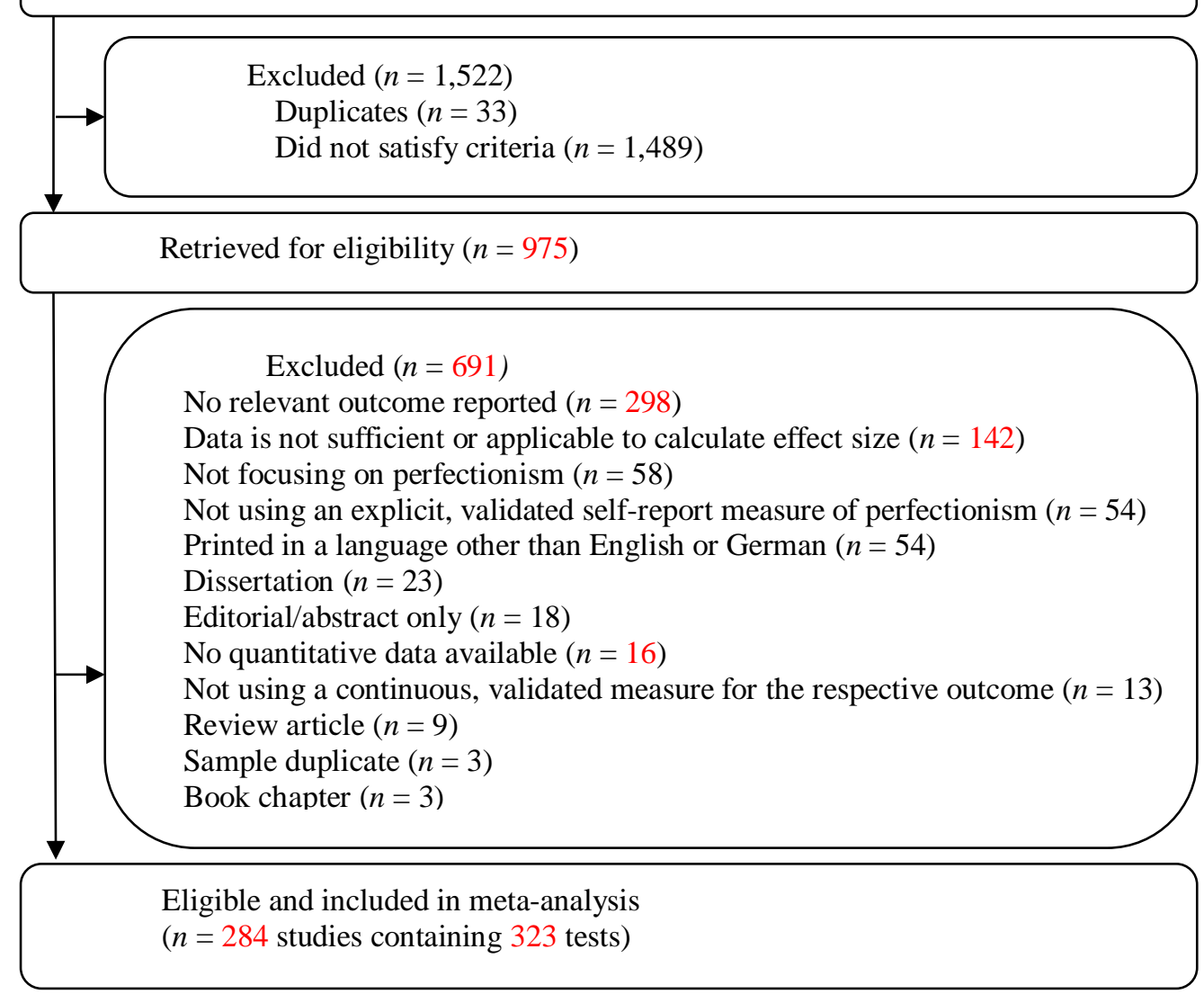

Figure 2. Process of study selection. 


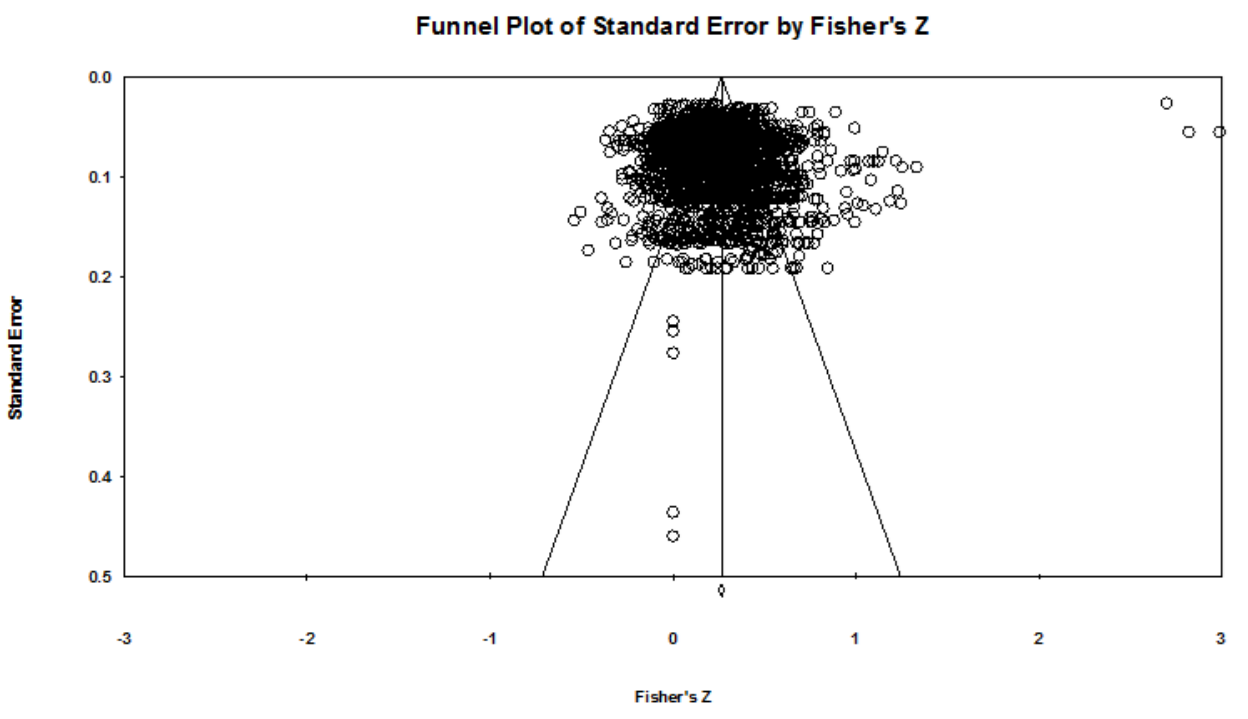

Figure 3. Funnel Plot of the Standard Error by the Effect Size Fisher's Z. 


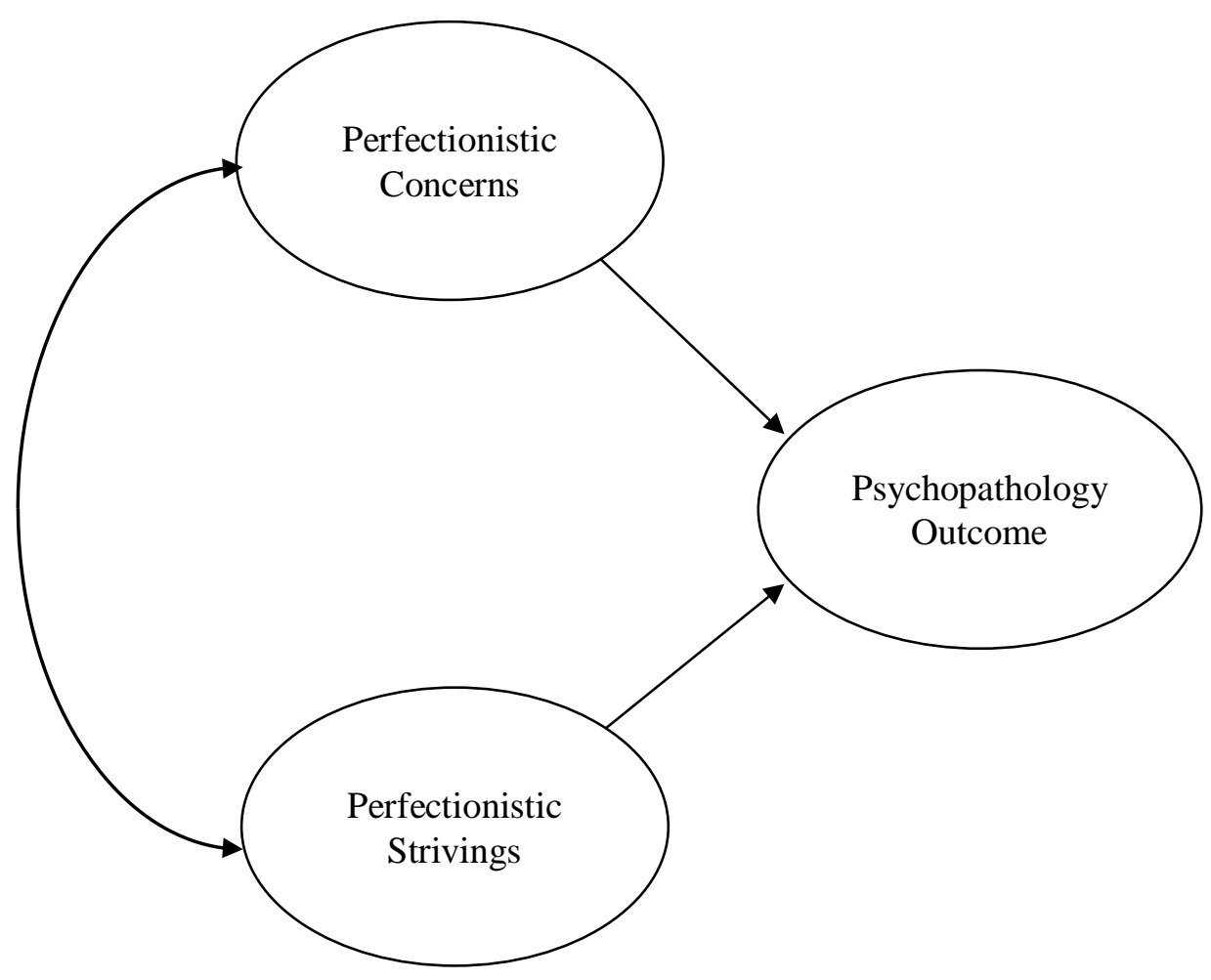

Figure 4. Diagram depicting generalized meta-analytic path model for effects of perfectionism dimensions on psychopathological outcomes. 